\title{
Kombination von Kiefergelenkersatz und Rekonstruk- tionen in der Unterkieferkorpus- oder Jochbeinregion mit erweiterten maßgefertigten Endoprothesen
}

\author{
Carl-Peter Cornelius, Michael Ehrenfeld, Gerson Mast, Oliver Driemel, \\ Anders Westermark, Christoph Leiggener
}

\section{Zusammenfassung}

Heutzutage gilt der totale alloplastische Kiefergelenkersatz bei Pathologien mit irreversiblen Form- und Dimensionsänderungen des Processus condylaris mandibulae als verlässliche Behandlungsoption. Bei Defekten, die über den Gelenkfortsatz und die Gelenkgrube hinaus weitere Unterkiefereinheiten und/oder die Jochbogen/ Jochbeinregion involvieren, lässt sich das Design individuell gefertigter Endoprothesen so gestalten, dass benachbarte Knochendefizite ausgeglichen werden können. Alloplastische Rekonstruktionen dieser Art kommen infrage, wenn autologe Rekonstruktionsverfahren mit erheblichem $\mathrm{Zu}$ satzaufwand verbunden oder nicht durchführbar sind. Während die anatomische Form und Konturgebung in ästhetisch und funktionell befriedigender Weise wiederhergestellt werden können, darf von einem alloplastischen Gelenkersatz mit Reduktion auf eine einfache Biomechanik von vornherein nicht erwartet werden, die Bewegungsabläufe und Interaktionen innerhalb des komplexen stomatognathen Systems vollkommen zu replizieren.
Combination of Temporo Mandibular Joint Replacement and Reconstructions in Mandibular Body or Zygoma Region with Extended, Custom-Made Endoprostheses

Nowadays total alloplastic mandibular joint replacement is considered to be a reliable therapeutic option for pathologies with irreversible changes in shape and dimensions of the mandibular condyle. For defects that involve further units of the lower jaw and/or the zygom or zygomatic region beyond the articular process and the glenoid fossa, individual prostheses can be so designed that neigbouring bone deficits are also compensated for. Alloplastic reconstructions of this type may be taken into consideration when autologous reconstruction procedures would be very time-consuming or even impossible. Although the anatomic form and contours can be reconstructed in an aesthetic and functionally satisfactory manner, one cannot expect from an alloplastic joint replacement limited to simple biomechanics that the motion sequences and interactions within the complex stomatognathic system will be completely replicated.

\section{Einleitung/Hintergrund}

Ein totaler alloplastischer Kiefergelenkersatz besteht obligat aus 2 Komponenten: einem der Gelenkpfanne (Fossa articularis) entsprechenden Gehäuse und einer Osteosyntheseplatte mit einem Aufsatz bspw. in Kugel- oder Walzenform, der dem Gelenkfortsatz einschließlich des Gelenkkopfs gleicht. Eine

OP-JOURNAL 2013; 29: 184-199

(c) Georg Thieme Verlag KG Stuttgart · New York DOI http://dx.doi.org/10.1055/s-0033-1351014

nach der Rekonstruktion mit einem autologen Gewebetransfer, einem alloplastischen Ersatz oder einer Kombination daraus. Die beiden Grundelemente einer Kiefergelenkendoprothese lassen sich bei Maßanfertigungen („Custom-made Device“) ausbauen, um assoziierte skelettale Defekte bis hin zum vollständigen Verlust der Unterkieferkontinuität zu schließen. Diese Möglichkeiten werden im Folgenden an exemplarischen Patientenbeispielen aufgezeigt.

\section{Kiefergelenke und Bewegungsabläufe}

Wie der Name sagt, stellen die Temporomandibular- bzw. Kiefergelenke (TM Joints) die Verbindung zwischen den beiden Dorsalenden der U-fömigen Mandibula und den Schläfenbeinen (Ossa temporalia) her. Die Gelenkpfannen befinden sich auf der Unterseite der Schläfenbeine und sind unmittelbar vor den äußeren Gehörgängen lokalisiert. Jede Pfanne wird von einem simsartigen Knochenvorsprung, dem Tuberculum articulare, nach vorne begrenzt. Die Ausdehnung und Zirkumferenz der Gelenkfläche (Facies articularis) ist deutlich größer als der walzenförmige Gelenkkopf, sodass ein erweiterter Bewegungsspielraum resultiert. Der Discus articularis, eine im Zentrum dünne faserknorpelige ovaläre Scheibe mit aufgewulsteten Rändern ist zwischen die gelenkführenden Flächen geschaltet. Der Diskus bedeckt die Gelenkwalze bis an den Scheitel wie ein Zeltdach. Da er in seiner gesamten Zirkumferenz mit der Gelenkkapsel verbunden ist, entsteht eine obere Gelenkkammer (zwischen Facies articularis und Diskus) und eine unterere Gelenkkammer (zwischen Diskus und Caput mandibulae). Dieses diskokapsuläre System ist durch die elastisch dehnbare bilaminäre Zone nach dorsal an der Schädelbasis befestigt. Nach vorne inserieren Fasern des M. pterygoideus lateralis mit einem oberen Kopf in den Vorderrand des Diskus und mit einem unteren Kopf direkt am Knochen in einer Grube
Bei komplexen Defekten, die neben dem Kiefergelenk weitere Unterkieferanteile und/oder die Jochbogen bzw. Jochbeinregion betreffen, stellt sich die Frage
[1]. Gelenkkopfaufsätze bzw. Condylar -ons an Rekonstruktionsplatten sung und sollten möglichst nicht ut eine Dauer von mehr als 2 Jahren in situ verbleiben.

Gelenkgrube ist notwendig, weil künstabgestützt sind, Gefahr laufen, in die mittlere Schädelgrube $\mathrm{zu}$ penetrieren 
vorne unterhalb der Gelenkwalze (Fovea pterygoidea). Die meisten Bewegungsabläufe im Kiefergelenk sind kombinierter Natur. Die Kaumuskeln kontrahieren sich nicht isoliert, sondern werden immer in einem koordinierten Zusammenspiel aller Kaumuskelgruppen unter Bezug auf Kontakte zwischen den Zähnen im Ober- und Unterkiefer (sog. Okklusion), bzw. die Stellung des Unterkiefers und die posteriore Gelenkführung (Kondylenbahn) aktiviert. Sofern eine Unterkieferkontinuität besteht, fungieren Kiefergelenke synchron im Sinne eines abhängigen Doppelgelenks und zwar bilateral symmetrisch (Öffnen bzw. Schließen, Vorschub, Retrusion) oder asymmetrisch (Laterotrusion, Mediotrusion). Bei Seitwärts- und/oder Einwärts- bzw. Mahlbewegungen reagiert die Gegenseite des Arbeitskondylus mit Ausgleichsoder Balancebewegungen. Bei Kieferöffnung und Schluss erfolgt eine DrehGleit-Bewegung in den Kiefergelenken. Während der initialen Dreh- oder Scharnierbewegung verbleiben Gelenkwalze und Diskus formschlüssig in der Fossa articularis. Zur weiteren Öffnung gleitet die Gelenkwalze mitsamt des Diskus unter dem Zug des unteren Pterygoideuslateralis-Kopfes nach vorne auf das $\mathrm{Tu}$ berculum articulare, womit sich die Rotationsachse nach vorne verschiebt und ihr Freiheitsgrad zunimmt. Die Schließbewegung ist durch einen Aktivitätsanstieg im oberen Pterygoideus-lateralis-Kopf gekennzeichnet. In Synergie mit den Elevatoren (Masseter/Pterygoideusmedialis-Schlinge und $M$. temporalis) sowie mit den Retraktoren (M. temporalis, posteriorer Teil, und suprahyoidale Muskulatur) wird infolgedessen der Discus articularis vorne gezügelt, was einen stabilisierenden Druck in Kaudalrichtung auf die Gelenkwalze ausübt und damit das dünne knöcherne Fossadach entlastet [2].

Die Kiefergelenke sind vermutlich die aktivsten Gelenke im menschlichen Organismus. Außer beim Sprechen und Kauen werden sie beim unbewusst ablaufenden Schluckakt von 1,5-2 Litern Speichel im 24-Stunden-Zyklus ca. 120150-mal eingesetzt. Ob der Kieferschluss beim Schlucken außer einer stabilisierenden Funktion für Zähne und Zahnstellung im Zusammenhang mit Gleichgewichtswahrnehmung, Raumorientierung und Körperhaltung steht, ist eine interessante, aber weitgehend unbeantwortete Frage [3].

\section{Verfahren zum Kiefergelenkersatz}

Historisch wurde über eine Reihe autologer Rekonstruktionsverfahren zum klinischen Kiefergelenkersatz - in Form von freien, nicht vaskularisierten oder - falls anatomisch dazu geeignet - mikrovaskulären Gewebetransfers - berichtet [4]:

- Metatarsus (Mittelfußknochen) des 2. Strahles und Metatarsophalangealgelenk

- kostochondrale Transplantate

- Beckenkamm

- Calvaria/Schädeldach

- Processus muscularis

- Schlüsselbein, Sternoklavikulargelenk

- Fibula/Fibulakopf

Bei chronisch entzündlichen Arthritiden mit Kiefergelenkbeteiligung oder im Anschluss an einen fehlgeschlagenen alloplastischen Kiefergelenkersatz haben sich autologe Transplantationsverfahren als grundsätzlich ungeeignet erwiesen, da mit großer Wahrscheinlichkeit erneut entzündliche Affektionen und Reankylosierungen auftreten [5].

Neben den Ersatzplastiken sind auch Distraktionsverfahren im Sinne einer Sliding-Osteotomie [6] bzw. einer Transport-Osteogenese [7] des Ramushinterrands nach kranial in die Fossa beschrieben.

\section{Indikationen zum alloplastischen Kiefergelenkersatz}

- Rezidivierende Ankylose mit ausgeprägter heterotoper Knochenneubildung - refraktär gegenüber anderen Behandlungsverfahren

- Höhenverlust des aufsteigenden Unterkieferasts (R. ascendens) mit massiver Malokklusion als Konsequenz von: - Entwicklungsstörungen/Fehlbildungen (z.B. hemifaziale Mikrosomie [HFM])

- idiopathische Kondylusresorption

- Trauma (z.B. Schussverletzungen)

- Tumoren (gutartig: Osteochondrom)

- Mitbeteiligung bei entzündlichen Arthritiden (z.B. rheumatoide Arthritis)

- multipel voroperierte Kiefergelenke/ Endstadium

Bei Patienten mit HFM und ausgeprägten Hypo- oder Aplasien des Kiefergelenkfortsatzes (Pruzansky Typ IIb/Typ III), die nach initial autologer Knochentransplantation infolge von Progression der Gesichtsasymmetrie einer erneuten Rekonstruktion bedürfen, wurden mit Cus- tom-made TMJ Total Replacement Devices befriedigende Resultate erzielt [810].

Essenziell für Langzeiterfolge ist der simultane Einbau des Gelenkkopfersatzes und einer passenden Fossa-Komponente. Ein alloplastischer Gelenkopfersatz ohne artifizielle Gelenkpfanne hat die Tendenz zu Knochenarrosionen in der Fossa und zur Penetration in die mittlere Schädelgrube (Lindquist et al. 1993, Westermark et al. 2006). Ein alleiniger alloplastischer Gelenkkopfersatz, bspw. ein Condylar Add-on auf einer Rekonstruktionsplatte, gilt daher als temporäre Lösung.

Die Indikationen zur Verwendung von TMJ Total Joint Replacement (TMJ-TJR) Devices für die 2 im Folgenden gezeigten Patientenbeispiele ergaben sich aus den unter Punkt 2 und 3 aufgelisteten Erkrankungsformen. Die Rekonstruktion eines Kiefergelenks mit einem alloplastischen Gelenkersatz ist immer als eine Ultima-Ratio-Behandlung in Endstadien der Erkrankung zu sehen, was unabdingbar einen absolut kritischen Entscheidungsprozess zur Voraussetzung hat.

\section{Kontraindikationen}

- Infektionen in der Implantationsregion

- Unverträglichkeiten oder Allergien gegen einen oder mehrere Materialbestandteile des TMJ-TJR

- vorausgegangene Bestrahlung

- Hypervalenz und Überfunktion der Kaumuskulatur (z.B. therapierefraktärer Bruxismus)

Konfektionierter versus individualisierter alloplastischer Kiefergelenkersatz

Zum alloplastischen Kiefergelenkersatz gibt es 2 Kategorien von TMJ-TJR-Implantaten:

- konfektionierte Systeme bzw. „Stock TMJ Replacement Systems “ und

- maßgefertigte Prothesen bzw. „Custom-made TMJ TJR-Devices“

Größe und Typen von Stock-Implantatsystemen orientieren sich an Durchschnittswerten, Proportionen und typischen Konfigurationen im Kiefergelenkbereich. In der Regel werden sie intraoperativ freihand an die gegebenen Verhältnisse angepasst. Zur Aufnahme der konfektionierten alloplastischen Gelenkpfanne wird es notwendig, den Knochen im Bereich von Gelenkpfanne und Tuberculum articulare passgenau zu reduzieren. Um Kongruenz im R. ascendens zu 
erzielen, kann es erforderlich werden, einen Knochensupport mit autologem Transplantatmaterial aufzubauen, denn die Metallplatten am Prothesenschaft sind zu stark ausgelegt, um sie anzubiegen.

Custom-made TMJ-TJR Devices sind patientenspezifische Implantate (PSI), die auf der Basis von CT-Datensätzen und mithilfe von Stereolithografie-(STL-)Modellen oder in einem komplett virtuellen Workflow exakt nach den individuellen anatomischen Verhältnissen gefertigt werden. Es ist naheliegend, dass sich STL-Modelle auch verwenden lassen, um Probleme bei der Anpassung von Stock-Prothesen zu antizipieren und Vorbereitungen zu treffen [12].

Im Schrifttum sind seit 1945 mind. 10 verschiedene Stock-Systeme zum kombinierten Ersatz von Fossa und Kondylus beschrieben worden. Diese Zahl steht in einem gewissen Widerspruch zur seltenen Anwendung von alloplastischen Kiefergelenksrekonstruktionen (Übersicht, s. [13]). Nach vorsichtiger Schätzung werden in der Bundesrepublik Deutschland deutlich weniger als 100 TMJ-TJR Devices pro Jahr implantiert. Weltweit sind nur noch wenige TMJTJR-Systeme von den Gesundheitsbehörden zertifiziert. Genaue Daten zur Anwendungshäufigkeit sind nicht publiziert. Die zurzeit meist verwendeten TJR-Implantate sind angeblich das konfektionierte Biomet (-Lorenz) TMJ Replacement System (Biomet Microfixation, Jacksonville, FL, USA) und das individualisierte TMJ Concepts Patient-Fitted Total Temporomandibular Joint Reconstruction System (TMJ Concepts, Ventura, CA, USA).

Das Biomet-Lorenz-System besitzt 9 verschiedene Prothesenschaftvarianten (3 Längen - small [45 mm], medium [50 mm], large [55 mm] in 3 Breiten narrow, standard, offset, jeweils für die rechte und linke Seite - sowie Fossagehäuse in 3 Größen zur Auswahl nach dem Baukastenprinzip für die bestmögliche Adaptation. Der sphärisch geformte Kondylenkopf besteht aus einer CobaltChrom-Legierung. Die Unterseite bzw. mediale Oberfläche des Prothesenschafts ist mit einem Titan-Plasma-Spray beschichtet. Die Fossaprothese ist ausschließlich aus Hochdruckpolyethylen (Ultra High Molecular Weight Polyethylene - UHMWPe) hergestellt, einem thermoplastischen Kunststoff, der für einen besonders leichten Lauf bei gerings- tem Abrieb sorgt. Gegenüber den natürlichen Verhältnissen verlagert das FossaDesign den Rotationspunkt des Kondylus nach kaudal, was auf der Bewegungsbahn bereits einer Translationsstellung entspricht und eine größere interinzisale Öffnung ermöglicht $[14,15]$.

Eine nunmehr 10 Jahre alte Vergleichsstudie zwischen Stock-TMJ-TJR-Implantaten (Christensen Prothese, TMJ Inc, Golden, Co, USA) und den Custom-made TMJ-Concepts-Prothesen attestiert den individualisierten Devices Überlegenheit nach subjektiven (Schmerzreduktion) und objektiven (z. B. maximale Interinzisaldistanz) Kriterien. Eine kürzlich veröffentlichte Studie konnte anhand der STL-Modelle von individuell geplanten TMJ-TJR allerdings zeigen, dass sich in 77\% der Fälle durchaus auch Stock-TMJProthesenkomponenten aus dem Biomet-Lorenz-System bei gleicher Passung hätten verwenden lassen [16]. Individuell angefertigte Kiefergelenkendoprothesen scheinen den im Vergleich zum konfektionierten Kiefergelenkersatz logistischen (s.u.) und finanziellen Mehraufwand (Materialkosten ca. $4500 €$ vs. ca. 9000 US\$) nur in Sonderfällen zu rechtfertigen.

Falls mit der Erkrankung im Kiefergelenkbereich erhebliche Deformationen oder Defektsituationen im benachbarten Unterkiefer und/oder im Gelenkgruben/ Jochbogenbereich verbunden sind, ist ein solcher Fall gegeben.

Immer wieder hervorgehoben wird die potenzielle intraoperative Zeitersparnis der Custom-made Endoprothesen, da ein Anbiegen der vorgeformten Devices natürlich entfällt und beim Einschrauben in der Regel sofort Primärstabilität erreicht wird [17].

\section{TMJ Concepts Patient-Fitted Total Temporomandibular Joint Reconstruction System}

\section{Design und Herstellungsprozess - technische Details}

Die von uns verwendete TMJ Concepts Patient-Fitted Temporomandibular Joint (TMJ) Reconstruction Prosthesis (TMJ Concepts, Ventura, CA) ist ein CAD/ CAM-gefertigtes Implantat zum Ersatz des kompletten Kiefergelenks.

Die Entwicklung des TMJ Concepts Device geht auf das Ende der 1980er-Jahre und die damalige Techmedia Endopro- these (Techmedia, Camarillo, CA, USA) zurück, die vorwiegend für mehrfach voroperierte Patienten mit mutilierter Anatomie der Gelenkregion konzipiert war. Die artikulierende Gelenkfläche der Fossa, bestehend aus UHMWPe, wird von einer individualisierten engmaschigen Gitterstruktur aus kommerziell reinem Titan getragen. Das Titanmesh überragt die Lateralfläche des Arcus zygomaticus und wird dort mit seitlich inserierten Schrauben befestigt. Die Schaftkomponente wird ebenfalls auf der Lateralfläche des Unterkiefers in der Korpus- oder Ramusregion angebracht. Der Schaft besteht aus einer Titanlagierung, während der Kondylenkopf aus Chrom-Kobalt-Molybdän aufgebaut ist.

Während des Herstellungsprozesses der TMJ-Concepts-Custom-made-Endoprothese wird dem Behandler ein auf Basis des CT-DICOM-Datensatzes hergestelltes Stereolithografie-(STL-)Modell des Gesichtsschädelskeletts zugesandt, das er zur OP-Simulation verwendet [5, 18,19-21]. Die Modellmandibula wird unter Berücksichtigung der Okklusion und der ästhetischen Konturgebung im Untergesicht positioniert und in dieser Stellung mit zahnärztlichem Silikon-Abdruckmaterial am Oberkiefer fixiert. Sofern nicht schon eine Defektsituation im Kiefergelenkbereich vorliegt, wird eine Resektion des Kiefergelenkfortsatzes (Kondylektomie) am Modell durchgeführt. Zur Anfertigung der Endoprothese geht das montierte STL-Modell an den Hersteller zurück. Auf dem STL-Modell wird unter Verwendung konfektionierter Wachsreplikate für den Kondylenkopf und die Fossa articularis (Gelenkfläche aus UHMWPe inkl.), Freihand eine Wachsmodellation der zu ersetzenden Knochen-/Gelenkpartien vorgenommen (Abb. 1 a). Vorweg müssen zur Einpassung des Fossagehäuses oftmals noch Radierungen am STL-Modell durchgeführt werden, wobei die abgeschliffenen Bereiche farbmarkiert werden. Zur stabilen Verankerung der gelenktragenden Schaftkomponente werden zwischen 7 und 10 Schraubenlöcher vorgesehen, die Fossakomponente erhält mindestens 4 Löcher zur Fixierung.

Die Maße und Dimensionierung des Wachsmodells werden dann in eine technische Zeichnung übertragen (Abb.1b) und die Daten zur Programmierung einer CNC (Computerized Numerical Control)-Fräse verwendet. Die in einem automatisierten Fräsprozess form- und maßgenau hergestellten 

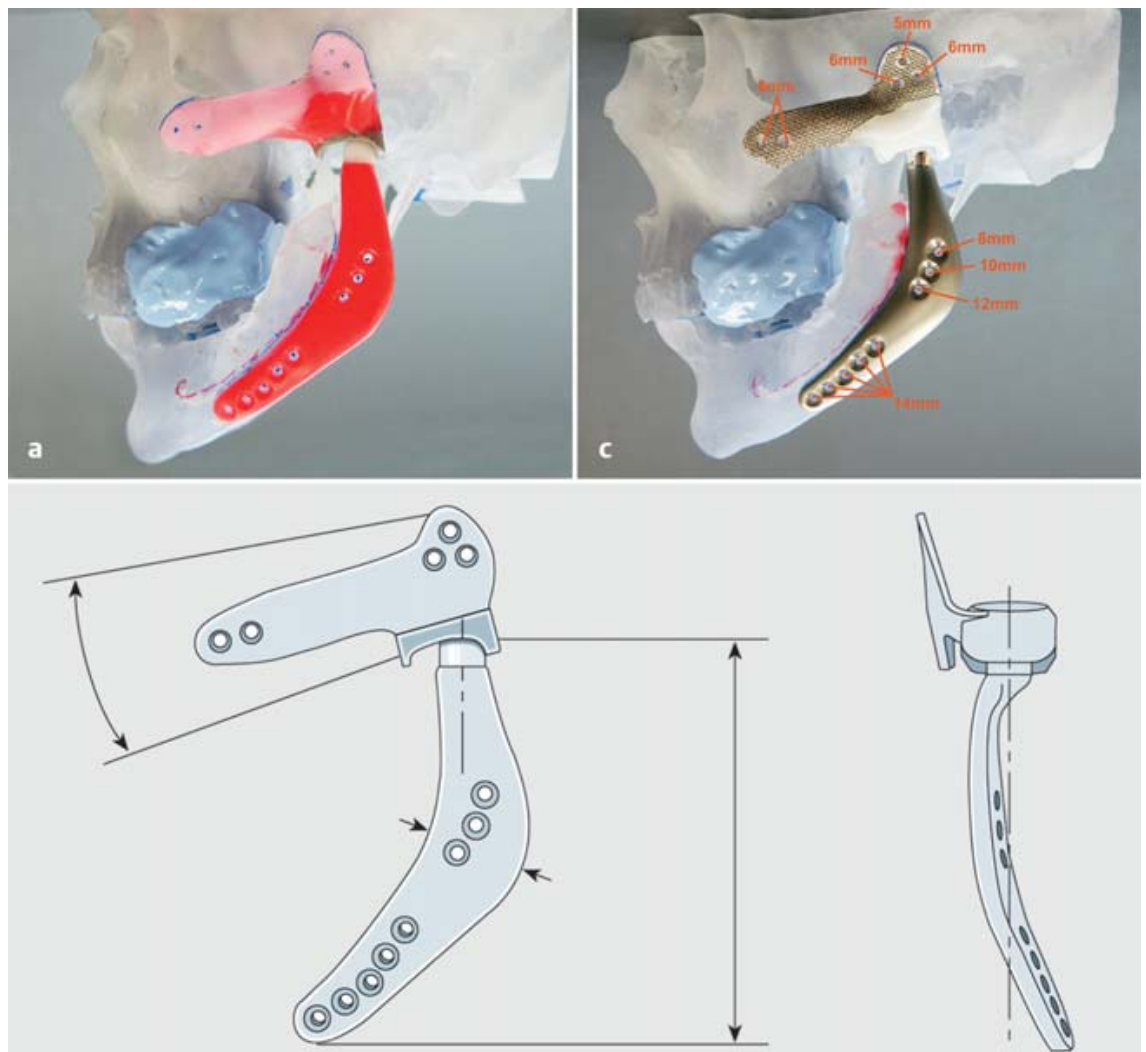

b

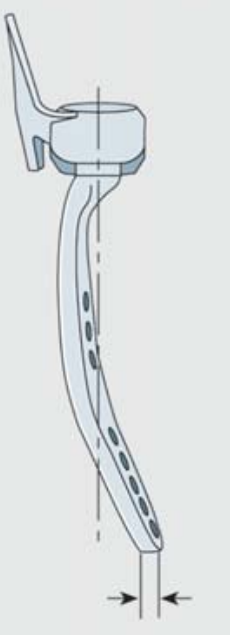

Abb. 1 a bis c Herstellungsprozess einer Kiefergelenkendoprothese - a Individuelles, aus CTDaten generiertes Stereolithografie-(STL-)Modell des Gesichtsschädelskeletts mit Wachsmodellation eines TMJ-TJR, Ersatz der Kiefergelenkspfanne extendiert um den Jochbogen, Kondylenkopf und R. ascendens; b technische Zeichnung; c CNC-gefrästes Endprodukt reponiert auf dem STL-Ausgangsmodell mit aufgedruckter Angabe der Schraubenlängen.

Werkstücke aus Titan und UHMWPe werden kontrolliert, zusammengefügt und erhalten ein Finish in Handbearbeitung.

Die fertige TMJ-TJR-Endoprothese wird in das STL-Modell reponiert und damit abschließend auf Passgenauigkeit überprüft (Abb.1c). Intraoperativ müssen die Form, Oberflächenkonturen und Position der Knochen im Gelenkbereich durch Resektion, Beschleifen bzw. Radierungen und Umstellung weitestmöglich in eine identische Konfiguration mit dem STL-Modell gebracht werden. Um eine gute Passform zu erreichen, können mehrfaches Nachschleifen und Einprobieren der TMJ-TJR-Komponenten nötig werden.

Bei knöchernen Ankylosen mit ausgedehnten Fusionen zwischen Gelenkfortsatz und Schädelbasis muss dieses herkömmliche Prozedere evtl. abgeändert werden. Um das Knochenlager für die Prothese genauer erfassen zu können, folgt bis dato das Planungs-CT erst auf einen initialen OP-Schritt mit Ostektomie und Remodellation zum Trismus-Release [22].

Mithilfe von intraoperativen Navigationstechniken oder auch mit im Selective Laser-Sintering-Verfahren hergestellten Cutting Templates/Resection Guides erscheint ein einzeitiges Vorgehen mit Umsetzung einer vorgeplanten Knochenresektion durchaus möglich.

\section{Operativer Zugang - \\ Kombination von 2 Wegen}

Die Insertion einer TMJ-TJR erfolgt in aller Regel über 2 getrennte Hautinzisionen und Dissektionsrouten, in der Absicht, auf Höhe des Ohrläppchens eine Weichgewebebrücke (Haut, SMAS [superficial muscular aponeurotic system], Parotis, Masseteranteile) zu belassen, durch die der Hauptstamm des Gesichtsnerven (N. facialis-CN VII) bzw. der Plexus intraparotideus zur motorischen Innervation der mimischen Muskulatur nach vorne führt (Abb.2a und b). Dazu werden die typischen Zugänge zur Kiefergelenkregion in der (Prä-)AurikulärSchläfen-Region und in der Sub-/Retromandibular bzw. periangulären Region modifiziert - und den Größenverhältnissen ausgedehnter Implantate entsprechend erweitert (Abb. 2 c c).

Während der Zugangsweg in der (Prä-) Aurikulär-Schläfen-Region zur Exposition des Bereichs um den Gelenkkopf, die obere Collumregion und die Fossa articularis dient, wird über die sub-/retromandibuläre Route die Lateralfläche des Unterkiefers vom Kieferwinkel bis in den unteren Teil des Collums dargestellt und subperiostal ein Verbindungstunnel zwischen beiden Wundhöhlen geschaffen.

Zur Insertion extendierter TMJ TJRs lassen sich die beiden Zugänge zusätzlich erweitern.

Zur Schonung der temporalen und wangenwärts (Rr. zygomatici) ziehenden Fazialisäste sowie des Fazialishauptstamms wird nach der präurikulären oder aurikulären Inzision subfaszial bzw. subperiostal präpariert. Nach der Hautdurchtrennung wird die Fascia temporoparietalis (mit einzelnen in die Galea aponeurotica einstrahlenden Muskelfasern - sog. M. epicranius) als Komponente des SMAS in Richtung auf die Temporallinie durchtrennt, um auf das oberflächliche Blatt (Lamina superficialis) der Fascia temporalis einzugehen und das SMAS nach ventral abzuschieben. Durch das transparente temporale Faszienblatt schimmert dann bereits der temporale Fettkörper (Temporal Fat Pad). Das Faszienblatt wird bogenförmig von vorne oben nach hinten unten entlang des Fettkörper-Ober- bzw. -Hinterrands inzidiert und der Jochbogenansatz freigelegt. Zur Exposition der Knochenstrukturen und der Kiefergelenkkapsel werden die Weichgewebeschichten als Einheit schließlich auf der Oberfläche des Fettkörpers und subperiostal über dem Jochbogen nach ventral abgeschoben (Abb. 2d). Bei diesem Prozedere liegen der N. facialis und seine oberen Äste ebenso wie der $\mathrm{N}$. auriculotemporalis und die Temporalgefäße immer außerhalb der Präparationsebene und werden nach vorne mobilisiert (Abb.2d). Die aurikuläre (ggf. auch transtragale) Inzision hinterlässt noch weniger sichtbare Narben [23]. Sie wird innen auf der dorsalen Traguskante platziert (Abb.2c), verläuft von dort nach dorsal um das Crus helicis und weiter entlang der He- 
a
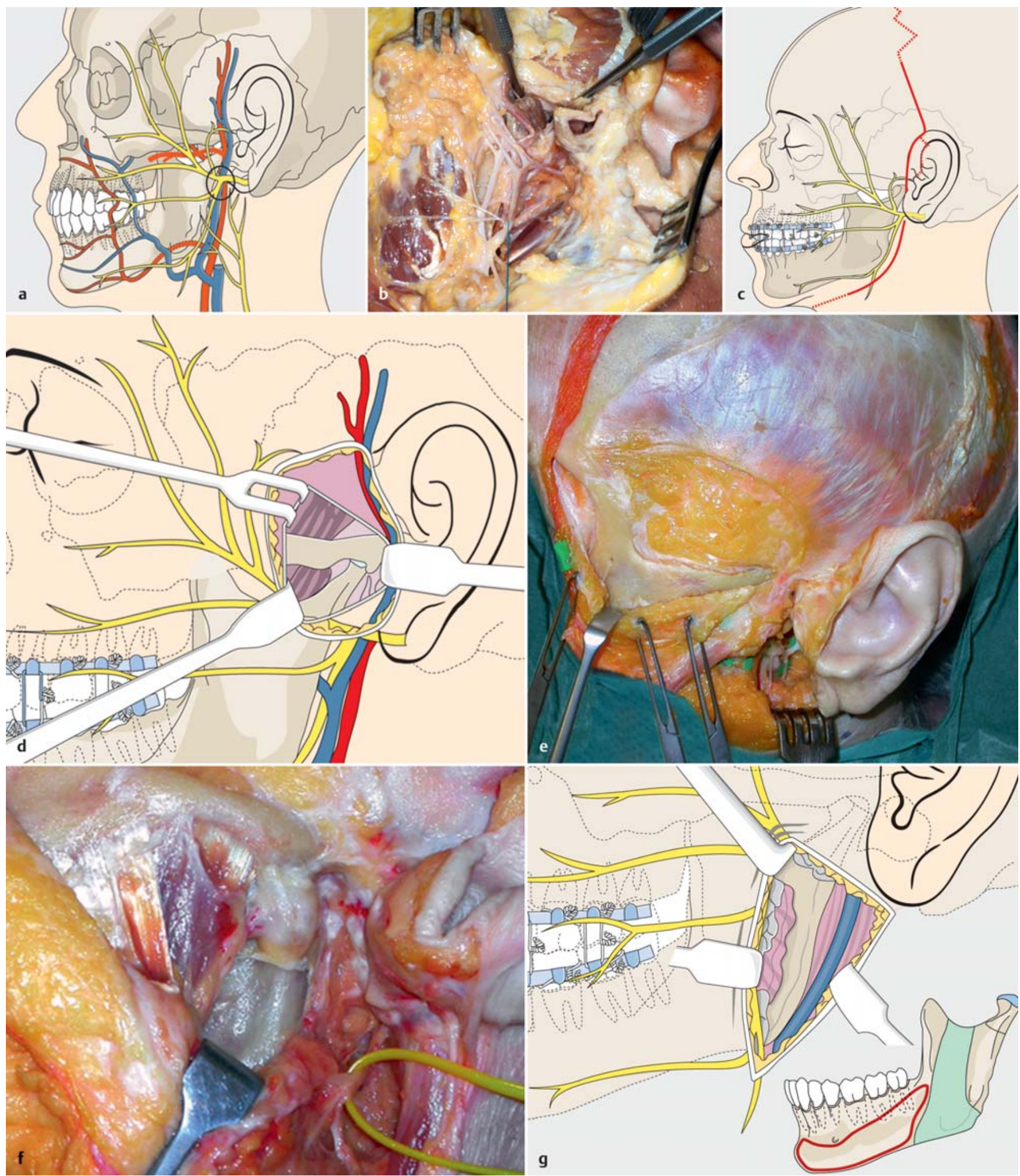

Abb. $\mathbf{2}$ a bis $\mathbf{g}$ Operativer Zugang zur Insertion von extendierten TMJ-TJR/KG-Endoprothesen über die Kombination von 2 Inzisionen bzw. Routen Schema und anatomische Grundlagen - a Chirurgische Anatomie mit der Temporomandibularregion mit N. facialis im Mittelpunkt; b korrelierendes anatomisches Präparat mit Darstellung von Gelenkwalze und Beziehungen zum Fazialisgeflecht, durchtrenntem äußeren Gehörgang, Gefäßen und Parotis; c kraniale und kaudale Hautinzisionen im Schema - Erweiterungen gepunktet; d Prinzip des kranialen Zugangs (s. Text); e anatomisches Präparat - kranialer Zugang: Oberfläche des temporalen Fat Pads und Darstellung des Jochbein-/Jochbogen-Ensembles, beachte: N. zygomaticotemporalis, Fazialisäste grün unterlegt; f anatomisches Präparat - kranialer Zugang: Darstellung von Kiefergelenkkapsel und oberer Gelenkfortsatzregion, Fazialishaupstamm mit gelbem Gummi-Loop nach kaudal abgedrängt; $\mathbf{g}$ Prinzip des sub-/retromandibulären Zugangs am aufsteigenden Unterkieferast bis auf Höhe des Gelenkfortsatzes. 


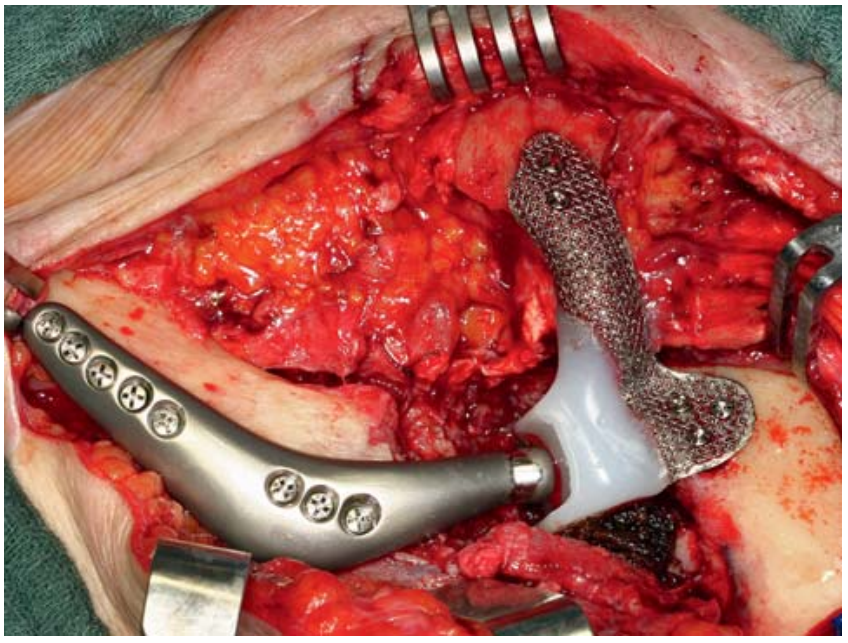

Abb. 3 Kontinuierliche Inzision und Wundkavität vom Unterkieferkorpus bis zur Schläfe nach totaler Parotidektomie (inkl. N.-facialis-Geflecht) und Resektion der Gelenkstrukturen zusammen mit dem Jochbogen und vorderen Gehörgang. Extendierte TMJ-TJR in situ.

lixkante bis kurz vor den Beginn der oberen Kurvatur und biegt in die behaarte Schläfenregion ab. Die aurikuläre Inzision umfährt das Innervationsgebiet des N. auriculotemporalis.

Der sub-/retromandibuläre Zugang orientiert sich an den Hautfalten direkt unterhalb bzw. um den Kieferwinkel (periangulär) und verläuft von dort aus nach kranial- und kaudalwärts (Abb.2g). Nach Inzision der Haut wird auf dem Platysma ca. $1 \mathrm{~cm}$ nach kaudal präpariert und der Muskel dort im Sinne einer Wechselschnittführung durchtrennt. Medial der oberflächlichen Halsfaszie wird der R./Plexus marginalis mandibulae des N. facialis (CN VII) sichtbar. Zusammen mit dem umgebenden Weichgewebe wird das Marginalis-Nervengeflecht nach kranial mobilisiert, bevor das Periost entlang des Unterkieferrands eingeschnitten und der M. masseter subperiostal in Richtung auf den Gelenkfortsatz abgeschoben wird. Bei Ausdehnung des Zugangs nach ventral in Richtung Kinn oder bis auf die Gegenseite müssen die Fazialgefäße ligiert und durchtrennt werden. Zur Erweiterung des Zugangs nach kranial bis unterhalb des Ohrläppchens wird das Platysma bis an sein über der Ohrspeicheldrüse liegendes Dorsalende verfolgt, wo es sich als SMAS fortsetzt. Bei der Eröffnung dieser Strukturen und Präparation um den Hinterrand der Drüse sollte der R. posterior des $\mathrm{N}$. auricularis magnus abgedrängt und geschont werden. Die Fascia parotideamasseterica überzieht die Drüsenoberfläche und den Ramushinterrand und wird in der Rinne dazwischen mitsamt dem Periost durchtrennt.
Das gesamte Jochbein/Jochbogen-Ensemble bis in der Bereich der Orbitaränder, die Stirn und die Schläfenregion wird freigelegt, indem die obere Inzision zur Bildung eines frontalen Skalplappens im Sinne eines Bügelschnitts nach kranial über den Vertex bis auf die Gegenseite verlängert wird (Abb. 2c, e und f).

Nur im seltenen Fall nach Tumorresektionen von Parotis und temporomandibulären Strukturen mit Opferung des N. facialis und seiner Äste ist die Präparation einer durchgehenden Wundkavität vom Unterkieferrand bis in die Schläfenregion zur Aufnahme einer extendierten TMJ TJR möglich (Abb. 3).

\section{OP-Sequenz}

Im Unterschied zu Osteosynthesen bei Frakturversorgungen des Unterkiefers oder bei der Überbrückung von Unterkieferdefekten mit Titanplatten dürfen Kiefergelenkendoprothesen nicht mit der Mundhöhlenschleimhaut oder mit Speichel in Kontakt kommen, damit die Grenzflächen gar nicht erst von einem bakterienhaltigen Biofilm bewachsen werden [24].

Werden transorale Umstellungsosteotomien (z.B. Le-Fort-I-Osteotomie im Oberkiefer oder z.B. sagittale Spaltung im kontralateralen R. ascendens des Unterkiefers), mit der transfazial/transkutanen Insertion von TMJ-TJRs kombiniert $[25,27]$, so müssen die beiden Operationsgebiete strikt voneinander getrennt bleiben, um eine Kontamination und spätere fremdkörperassoziierte Infektion zu vermeiden.
Die OP-Sequenz beginnt entweder mit den transoralen Schritten oder aber extern mit dem transfazialen Eingriff. Beim Start der Operation in der Mundhöhle werden alle auf diesem Wege möglichen OP-Teilschritte abgeschlossen und ggf. noch eine mandibulomaxilläre Immobilisation durchgeführt. Anschließend wird die Gesichts-Hals-Haut erneut desinfiziert und das Operationsgebiet neu steril abgedeckt. Zusätzlich wird die Lippen-, Kinn- und Naseneingangsregion mit einer transparenten Inzisionsfolie (z.B. Tegaderm, 3M) versiegelt. Bei Beginn der Operation mit Insertion der TMJ-TJR von extern muss auch dieser Teilschritt komplett, d.h. einschließlich Wundverschluss, zu Ende geführt werden.

Bei Extension des Endoprothesenschafts bis in den Kinnbereich oder auf die Unterkiefergegenseite kann eine sagittale Spaltung des R. ascendens, anders als sonst üblich, auch von extraoral durchgeführt werden (Abb. 4).

\section{Planung patientenspezifischer totaler alloplastischer Kiefergelenkersatz - TMJ TJR}

Deformitäten und Defekte in der Temporomandibularregion gehen meistens mit Okklusionsstörungen, dentoalveolären Veränderungen, Lageanomalien und Formabweichungen des Unterkiefers (v.a. Asymmetrien) sowie Anomalien des Gesichtsskeletts (z. B. Über- oder Unterentwicklung) einher [27].

Die morphologischen Veränderungen und Funktionsstörungen im Kiefergelenksbereich sind entweder die Ursache von dentalen und skelettalen Anomalien oder umgekehrt das Resultat derartiger Veränderungen, was als natürlicher Kompensationsversuch zu werten ist. 


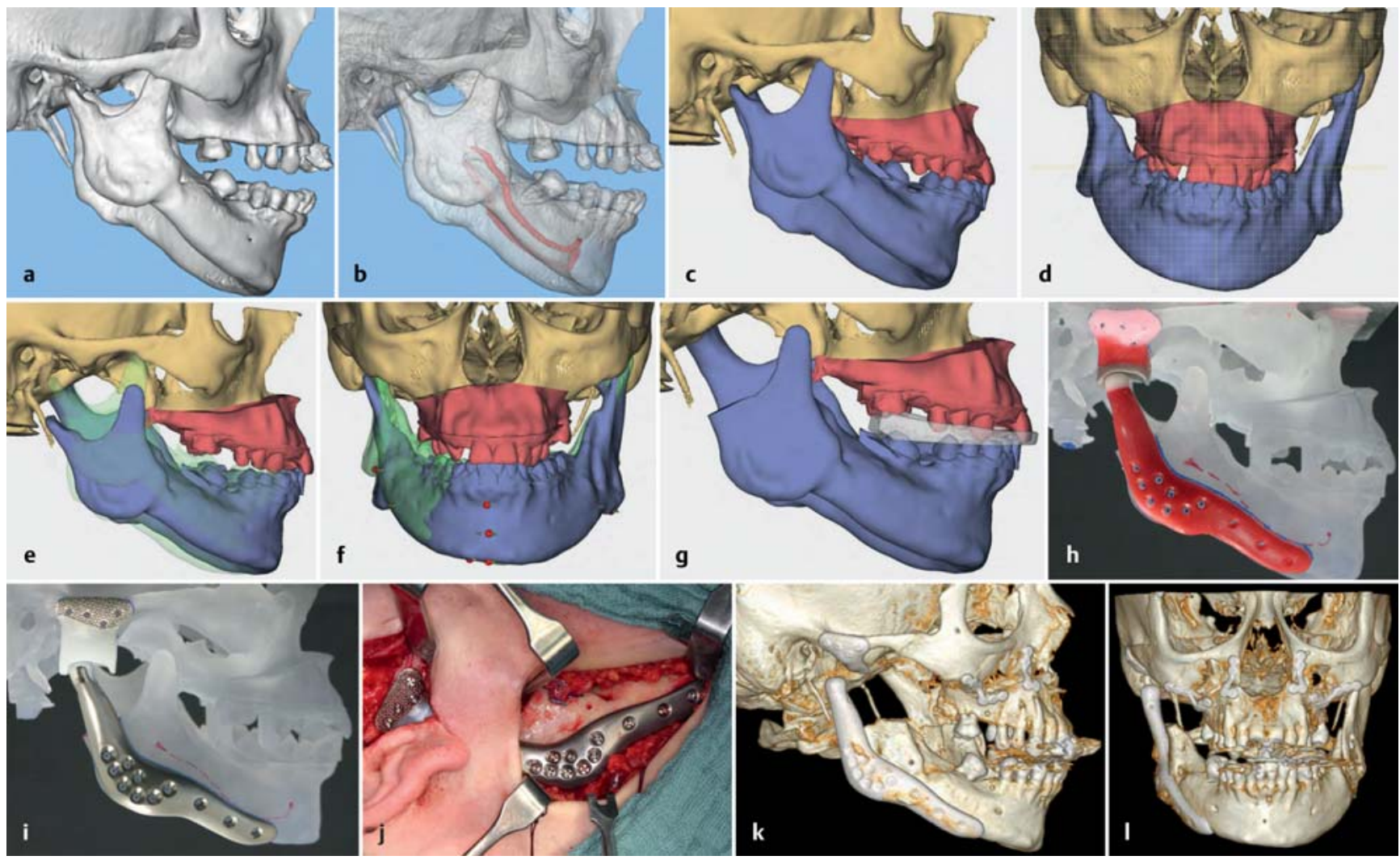

Abb. $\mathbf{5 a}$ bis I Virtuelle Planung einer Umstellungsosteotomie im Oberkiefer (Reoperation nach bilateraler sagittaler Spaltung im Unterkiefer) in Kombination mit einer in den Unterkieferkorpusbereich extendierten TMJ-TJR rechts - Status nach 3-maliger Voroperation des Kiefergelenks rechts mit Kondylusdefizienz - a Fusioniertes Gesichtsschädelskelett-Kiefermodell; b Topografie der Mandibularkanäle (rot) in Transparenzdarstellung; c und $\mathbf{d}$ Oberkiefersegment (rot) nach Le-Fort-I-Osteotomie; e und $\mathbf{f}$ Vorverlagerung und Rotation des Oberkiefersegments um eine Sagittalachse („Roll“) zur Okklusionseinstellung (seitlich offener Biss beidseits in Vorbereitung einer prothetischen Neuversorgung), Nivellierung der Kauebene mit Derotation des Unterkiefers; $g$ Skelettbezüglich korrekt ausgerichteter „mandibulomaxillärer Block“, virtueller definitiver interokklusaler Splint und resezierter Restkondylus; $\mathbf{h}$ STL-Hybridmodell mit umgestellten Kiefersegmenten und Wachsmodellation einer bis in den Unterkieferkorpusbereich extendierten TMJ-TJR; i Kontrolle der patientenspezifischen TMJ Concepts TMJ-TJR auf dem STL-Hybridmodell; j Extendierte TMJ-TJR intraoperativ; $\mathbf{k}$ und I postoperative 3-D CTs - L-förmige Miniosteosyntheseplatten über den Pfeilerstrukturen in der Le-Fort-I-Ebene und TMJ-TJR rechts in situ, ergänzend zur Planung wurde der R. ascendens links reosteotomiert (sagittale Spaltung), um eine Distraktion im Kiefergelenk auszugleichen. Der Hochdruckpolyethylenanteil der Fossa bildet sich röntgenologisch nicht ab.

Neben einem alloplastischen TMJ-TJR zur Behebung ansonsten irreparabler Kiefergelenkdestruktionen (bspw. idiopathische Kiefergelenkresorption, Kondylushypo- und -hyperplasien, Osteoarthrose, kongenitale Deformation oder Nichtanlage, Ankylose) werden in integrativen Behandlungskonzepten deshalb operative Korrekturen des Unter- und/oder Oberkiefers in die Therapie einbezogen. Da im Herstellungsgprozess patientenspezifischer TMJ-TJRs ohnehin CT-DICOM-Datensätze und ein STL-Modell benutzt werden, bietet sich die OP-Simulation von Umstellungsosteotomien in einem computerassistierten virtuellen „workflow" mit 3-D-Visualisierung an (Abb.5). Die Repräsentation der Zahnreihen und Okklusalflächen ist jedoch selbst in Feinschicht-CTs mit Slice Increments von $0,6 \mathrm{~mm}$ zur Okklusionseinstellung nicht präzise genug.
Um die Kiefersegmente exakt in die gewünschte neue Position bringen zu können, werden daher die hoch detaillierten okklusalen Oberflächen von Kiefermodellen aus Hartgips eingescannt und mit dem CT-Bilddatensatz (Abb.5a und b) fusioniert (Methodik z.B. nach Zizelmann et al. $[28,29])$. Am Bildschirm werden an diesem zusammengesetzten Schädelskelett-Kiefer-Modell zunächst die Osteotomien angelegt und die Kiefersegmente vom umgebenden Skelett separiert (Abb. 5c und d). Darauf folgt die Einstellung der definitiven Okklusionsposition, womit die zahntragenden Segmente im Ober- und/oder Unterkiefer zugleich verschoben und in einem „mandibulomaxillären Block“ [30] arrangiert werden. Wie bei einer bignathen bzw. bimaxillären Standardumstellung, d.h. Le Fort I plus bilaterale sagittale Spaltung der aufsteigenden Unterkieferäste, wird dieser Block durch Translations- und Ro- tationsbewegungen in den 3 Raumebenen ausgerichtet, bis harmonische Verhältnisse im Gesichtskelett hergestellt sind. Durch einen fehlenden Unterkieferkondylus und den daraus resultierenden Möglichkeiten zur Reposition des Unterkiefers in Kombination mit einer Le-FortI-Osteotomie ist eine analoge Situation zur bignathen Standardosteotomie gegeben. Falls die Bewegungen über den Spielraum des kontralateralen Kiefergelenks hinausgehen, muss der Unterkieferramus auf dieser Seite noch einer sagittalen Spaltung unterzogen werden (Abb.5e und f). Die zur intraoperativen Einstellung der Segmente bzw. des Unterkiefers notwendigen interokklusalen Splints (Anfangs-/Zwischen- und definitive Wafer) werden virtuell entworfen (Abb.5g) und später im selektiven Laser-Sintering-Verfahren produziert. Eine im Kondylusbereich notwendig werdende Resektion lässt sich ebenso einfach 
virtuell vornehmen wie die Umstellung der Segmente. Nach Beendigung der virtuellen Operationssimulation und Abschluss des Splint-Designs wird aus den digitalen Datensätzen ein STL-Hybridmodell der Sollsituation gebaut. Darauf wird von Hand die Wachsmodellation der TMJ-TJR-Endoprothese durchgeführt (Abb. 5h) und schließlich die Passgenauigkeit der patientenspezifischen Endoprothese kontrolliert (Abb.5i), bevor diese zum klinischen Einsatz kommt (Abb. 5j bis I).

Ein vollständiger virtueller Workflow ohne manuelle Zwischenschritte bzw. „analoge Lücken“ ist von der Firma TMJ Concepts bisher noch nicht realisiert.

Einen Transfer digitaler Planungs- und Designdaten ohne Umwege in Titanimplantate, die im additiven Printing-Verfahren hergestellt werden, offeriert die Firma Xilloc Medical BV, Maastricht, beim Unterkiefer/TMJ-TJR allerdings nicht zusammen mit dem obligaten [1] Fossaersatz (www.xilloc.com/patients/ stories/total-mandibular-implant).

\section{Extendierte TMJ-Endoprothesen}

Bei der Mehrzahl der inserierten TMJTJRs handelt es sich um streng auf die Kondylus/Fossaregion limitierte Implantate, für die gute Langzeiterfolge über 15 Jahre und mehr beschrieben sind [31].

Seit einigen Jahren mehren sich Fallberichte über extendierte TMJ-TJRs, mit denen Knochendefekte oder Knochendefizite im angrenzenden Unterkiefer überbrückt oder ausgeglichen wurden, bspw. bei HFM $[9,10]$.

Hier werden die Planung extendierter TMJ-TJRs, die operative Vorgehensweise sowie die klinischen und radiologischen Ergebnisse kasuistisch bei 2 Patientinnen aufgezeigt.

Fall 1 - Osteosarkom des Unterkieferkorpus/Kieferwinkels im Kleinkindesalter

Eine inzwischen 21 Jahre alte Patientin stellte sich mit dem Wunsch nach weiteren funktionellen und ästhetischen Verbesserungen wieder vor, nachdem bei ihr im Kleinkindesalter von anderen Behandlern wegen eines Osteosarkoms eine bis in die Prämolarenregion reichende Mandibulektomie links mit Exartikulation des Kiefergelenks durchgeführt worden war (Abb.6a). Im Alter von 9 Jahren war zur Unterkieferersatz- plastik bereits ein revaskularisiertes Beckenkammtransplantat verpflanzt worden (vgl. [32,33]), wobei der Weichgewebeverlust in der Wange (Masseter/ Pterygoideus-medialis-Schlinge) nicht aufgefüllt wurde. Die Knorpel-KnochenManschette von der Crista iliaca des Transplantats war in der Hoffnung, damit ein Wachstum $\mathrm{zu}$ induzieren, auf das gelenknahe Transplantatende transponiert worden. (Abb.6b bis c1). Zum Zeitpunkt der Konsultation bestand eine Asymmetrie im Unter- und Mittelgesicht durch eine großflächige Einziehung in der Präaurikular-, Wangen- und Hemimandibularregion links, eine Schrägstellung und Ptose der Unterlippe nach links, eine Deviation der Kinnmitte nach links mit frontal offenem Biss sowie eine leicht erweiterte Lidspalte (,increased scleral show“) links, verursacht durch eine Fazialisschwäche (Abb. $\mathbf{6 d}$ bis f).

Es fiel die Entscheidung, das Kiefergelenk, die Hemimandibel und die Jochbogen/Jochbeinregion mit extendierten Komponenten einer alloplastischen TMJTJR aufzubauen. Durch Überkonturierung der Ausleger zum Unterkiefer und Jochbein sollte zugleich das Volumendefizit der Weichgewebe kompensiert werden. An 3-D-reformattierten CTs war das skelettale Defizit und die Defektsituation detailliert einzuschätzen (Abb. $\mathbf{6 g}$ bis j). Ein Wachstum des revaskularisierten Beckenkammtransplantats hatte offensichtlich nicht stattgefunden. Bei weitgehender Dimensions- und Formkonstanz war es nur zu einer Lageänderung (Relokation) nach anterior mit Größenzunahme des Defekts im temporormandibulären Übergang gekommen. Als Ursache der Deformation und Hypoplasie im Jochbein-Jochbogenbereich wurde hingegen eine Wachstumshemmung vermutet. Die Planung der Endoprothesen erfolgte unter Abgleich mit einartikulierten Kiefermodellen per Hand an den CT-basierten STL-Modellen (Abb.6k und I): nach einer paramedianen Osteotomie auf Prämolarenhöhe links wurde der Unterkiefer rechts um eine Vertikalachse derotiert, um eine schlüssige Okklusionsbeziehung im Seitenzahngebiet $\mathrm{zu}$ erreichen. Die neue Unterkieferposition wurde mit seitlich aufgeschraubten Miniplatten eingefroren. Das Beckenkammsegment links wurde lateralwärts reponiert und in dieser Stellung über eine lingual angebrachte Miniplatte eingefroren. Probatorisch wurde zusätzlich der frontal offene Biss durch eine Blockosteotomie (Regio 35 bis 42) und Anhebung des Segments ge- schlossen. Die Schaft-Gelenkkopf-Komponente des TJRs inkorporierte einen bis in den Korpusbereich der Gegenseite reichenden Mandibularbogen, der zur Stabilisierung des Gesamtkonstrukts an nativem Knochen endständig 6 Schraubenlöcher aufwies. Die Außenkonturen des Unterkieferkorpus wurden von der rechten Seite des vorbereiteten STL-Modells gespiegelt.

Zum Aufbau des „Wangenknochens“ reichte der Ausleger der Fossakomponente bis in den Bereich des Jochbeinkörpers und der Crista zygomaticoalveolaris. Dabei besaß die laterale Oberfläche der Extension in Fortsetzung der Gelenkgrube eine Hochdruckpolyethylen-(UHMWPe-)Beschichtung.

Die Exposition über einen koronaren und erweiterten submandibulären $\mathrm{Zu}$ gang sowie die anschließende Insertion der Endoprothesenkomponenten verliefen unproblematisch (Abb. $\mathbf{6 m}$ ). Von der anterioren Blockosteotomie wurde abgesehen, um die Vaskularisation des Segments nicht zu gefährden. Die Hochdruckpolyethylen-Fossa ist zwar dorsal mit einer längeren Lamelle ausgestattet, die Luxationen des Gelenkkopfes nach posterior verhindern soll. Intraoperativ war aber bereits festzustellen, dass durch die fehlende Masseter/Pterygoideus-Schlinge keine ausreichende Zugwirkung in dorsokranialer Richtung mehr vorhanden war, um den Gelenkkopf in der Grube zu halten. Zur Zügelung wurde daher ein Goretex-Streifen (WL Gore \& Associates, Flagstaff, AZ, USA) aus der Korpusregion links bis in den Kalottenbereich über der Temporallinie mithilfe von 2 Miniplatten aufgespannt (Abb.6n). Die postoperative Wundheilung und Integration der extendierten TMJ TJR war regelrecht (Abb.6o bis q). Eine Traktionsparese des N. facialis war innerhalb von 3 Monaten bis auf den Ausgangsbefund rückläufig. Die Kieferöffnung ist mit einer Interinzisaldistanz von $35 \mathrm{~mm}$ seit nunmehr 4 Jahren nahezu uneingeschränkt. Translationsbewegungen sind nicht möglich, dementsprechend sind Protrusions- sowie Laterotrusons- und Mediotrusionsbewegungen aufgehoben bzw. erheblich kompromittiert. Nach 2-maliger Fettgewebeaugmentation (Coleman Lipostructure $[34,35]$ ) im Wangenbereich erscheint das ästhetische Ergebnis trotz der weiterbestehenden Fazialisschwäche akzeptabel (Abb. $6 \mathbf{r}$ und s). 


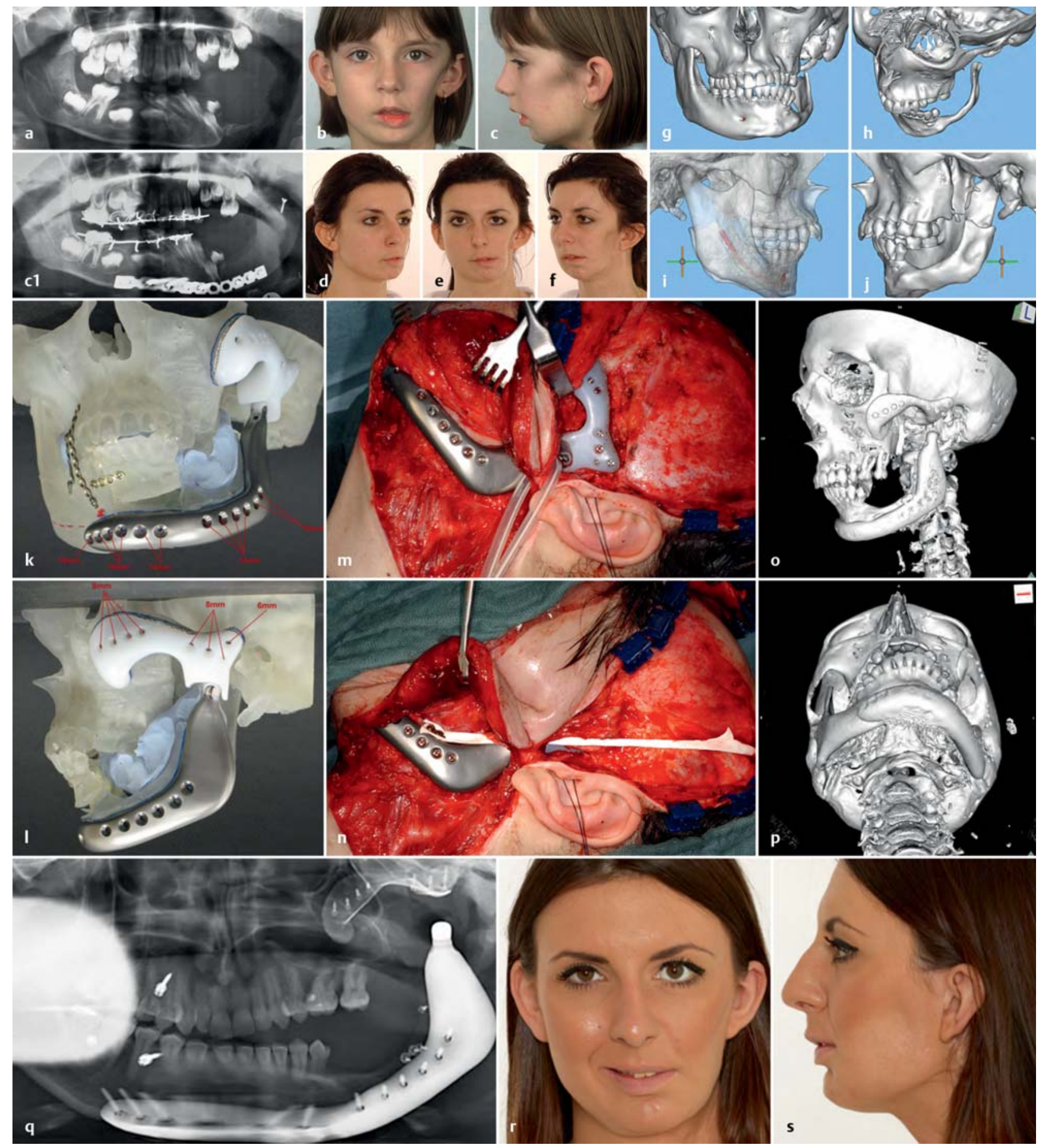

Abb. $\mathbf{a}$ bis $\mathbf{s}$ Mandibulektomie links mit Exartikulation des Kiefergelenks im Kindesalter wegen eines Osteosarkoms - a Panoramaröntgenschichtaufnahme (PSA) im Alter von 7 Jahren mit Unterkiefer/Kiefergelenkdefekt links; b und c Zst. nach Unterkieferersatzplastik mit einem revaskularisiertem Beckenkammtransplantat im Alter von 9 Jahren, Fazialisschwäche links; c1 PSA wenige Tage nach Beckenkammtransplantation, Drahtbogen-Schienen noch in situ, beachte: Schraubenfixation der Knorpel-Knochen-Manschette am Dorsalende des Transplantats; $\mathbf{d}$ bis $\mathbf{f}$ Gesichtskonturen bei Wiedervorstellung mit 21 Jahren; g bis i 3-D-CTs zur Evaluation der Knochendefizienzen und des Defekts im Kiefergelenksbereich; $\mathbf{k}$ und I STL-Modell nach Umstellung/Reposition des Unterkiefers und extendierter TMJ-TJR; $\mathbf{m}$ Endoprothesen-Komponenten intraoperativ nach Schraubenfixierung; $\mathbf{n}$ Zügelung mit Goretex-Streifen als Schutz vor Luxation des Kiefergelenkkopfs; o bis q 3-D-CTs und PSA 6 Tage post OP (MMF-Schrauben noch in situ); $\mathbf{r}$ und $\mathbf{s}$ weitgehend symmetrische Gesichtskonturen und Weichgewebevolumenverhältnisse im Alter von 25 Jahren (weitere Einzelheiten s. Fallbeschreibung im Text). 
Fall 2 - Kontinuierlicher Verlust des Unterkiefers im Korpus/Ramusbereich beidseits einschließlich der Kiefergelenke - SAPHO-Syndrom?

Nach langer Vorgeschichte und Behandlungspause suchte eine 59-jährige Patientin die Klinik wieder auf. Bis auf ein anteriores Teilstück in der Symphyse bzw. Kinnregion (Abb.7a) hatte sie im Laufe von 20 Jahren alle übrigen anatomischen Untereinheiten der Mandibula im Rahmen einer progredienten Osteolyse verloren. Das ursächliche Krankheitsgeschehen war immer wieder anders interpretiert worden - zuerst als akute Osteomyelitis, da die ersten Symptome in Form von Schmerzen, perimandibulärer Schwellung und Sensibilitätsstörungen im Anschluss an die Entfernung des unteren Weisheitszahns rechts (48) aufgetreten waren. Nach mehreren Dekortikationen, weiteren Zahnextraktionen und fortschreitenden Osteolyse wurde aufgrund der histologischen Befunde eine unifokale Langerhans-Histiozystose vermutet. In späterer Gesamtschau und nach weiteren feingeweblichen Untersuchungen wurde daraus eine chronisch progrediente Osteomyelitis. Der lokoregionale Prozess mit der Osteolyse hatte zwischenzeitlich beide Unterkieferhälften erfasst, wobei zuerst das Kiefergelenk rechts, später auch links von der Auflösung betroffen war.

Alle bisherigen Behandlungsversuche waren vergeblich gewesen.

Hauptbeschwerde bei der Wiedervorstellung war nicht etwa der Verlust der Kaufunktion, sondern die Verlegung der oberen Atemwege mit Apnoen beim Schlafen in Rückenlage. Aus diesem Grunde hatte die Patientin ihre Nächte während der letzten Jahre aufrecht sitzend in einem Stuhl zugebracht und immer nur kurze Schlafphasen gefunden. Da sie unfähig war, Nahrungsbestandteile oral zu zerkleinern, nahm sie nur noch Flüssigkeiten und Speisen in Breiform zu sich. Eine nennenswerte Sprechstörung war nicht feststellbar.

Klinisch imponierte ein weit zurückliegendes Untergesicht mit in allen Richtungen mobiler Kinnpartie, eine weit nach hinten gesunkene Zunge, die mit dem Rest der Kieferspange manuell nach vorn gezogen werden konnte, und eine aktiv eingeschränkte Kieferöffnung.

Im Vorfeld der Operationsplanung wurde in der interdisziplinären Reevalution von Vorgeschichte, Krankheitsverlauf, histologischen und laborchemisch-immunologischen (z. B. HLA B-27-) Befunden retrospektiv die Verdachtsdiagnose eines SAPHO-Syndroms gestellt. Diese Akronym setzt sich aus den Initialen der Hauptsymptome einer seltenen osteoartikulären Krankheitsentität zusammen: Synovitis, Akne, palmoplantare Pustulose, Hyperostose und Osteitis. Die genaue Ätiologie ist unbekannt, wahrscheinlich spielen gleichermaßen genetische, infektiöse und immunologische Komponenten eine Rolle. Die Hautmanifestationen stehen lange im Vordergrund, die Akne befällt überwiegend den Gesichts- und Nackenbereich, der Unterkiefer soll in ca. $10 \%$ der Fälle in Form einer Hyperostose mit Ankylosierung oder von Osteoysen betroffen sein. Diagnostische Schwierigkeiten ergeben sich daraus, dass die Symptome häufig asynchron auftreten. Im Falle unserer Patientin wurde eine Hyperostose der Sternoklavikulargelenke und rezidivierend auftretende psoriasiforme palmoplantare Pustulose erst im Nachhinein in die diagnostischen Überlegungen einbezogen [36-39]. Die Behandlung von SAPHO ist symptomatisch, eine kurative Therapie gibt es bisher nicht. Bei Kiefergelenksankylosen mit Öffnungseinschränkung im Gefolge von SAPHO wird vermutlich schneller mit chirurgischen Maßnahmen reagiert $[37,39]$ als im Fall einer Osteolyse.

Die Gründe, die fehlenden Unterkiefereinheiten in unserem Fall mit einer zusammenhängenden [40] oder 2 separaten extendierten Endoprothesen zu rekonstruieren, waren 2-fach: die unbekannte möglicherweise systemische Ätiologie der Osteolyse, die einen Knochentransfer unsicher und fragwürdig machte sowie keine Optionen zur Entnahme von vaskularisierten Fibula-, Beckenkamm- oder Scapula-Transplantaten nach den Angiografiebefunden. Vorgaben für die Planung des alloplastischen Unterkieferersatzes - bei Teilbezahnung ausschließlich am STL-Modell durchgeführt - waren der Erhalt des Restunterkiefers anterior und dessen Integration in das Metallkonstrukt zum Ersatz der Mandibulaanteile nach posterior. Bei der Positionierung dieses symphysealen Restknochenstücks musste ein Kompromiss gefunden werden. Zum einen musste es weit vorne eingestellt werden, um die Zunge und das Hyoid über ihre Muskelinsertionen an diesem Knochen wieder in eine möglichst physiologische Position zu bringen und die
Atemwegsobstruktion im velopharyngealen Übergang zu beseitigen. Im Gegensatz dazu war eine sehr weite Vorverlagerung mit der großen Gefahr verbunden, den Weichgewebemantel durch ein entsprechend langgestrecktes Implantat zu überdehnen und eine Exposition zur Mundhöhle und/oder durch die Hautoberfläche zu riskieren.

Damals wurde in Ermangelung einer Vorhersage der Veränderungen im oberen Atemwegsbereich durch computerassistierte Simulation (heute erscheint das näherungsweise möglich - [41]) die optimale Position schließlich durch Vorziehen des Knochenstücks in Rückenlage an der Patientin ermittelt und auf das STL-Modell übertragen (Abb. 7 b und c).

Während sich das Standarddesign der Fossakomponenten beibehalten ließ, bedeutete die Konstruktion der Mandibulakomponente Neuland. Da mit einer weiteren Progredienz der Osteolyse gerechnet werden musste, war es naheliegend, eine durchgehende Titan-Neomandibula zu gestalten, damit für alle Fälle ein stabiler In-toto-Bogen mit Resuspensierungsmöglichkeit (,frontal bumper") für die Zungenmuskulatur zur Verfügung stehen würde (Abb. $\mathbf{7} \mathbf{d}$ bis $\mathbf{g}$ ). Die Dimensionierung der Neomandibula entsprach einem mäßig atrophen Unterkiefer ohne scharfkantige Übergänge, sie besaß kleine Koronoidfortsätze zur Refixierung mit der Temporalismuskulatur. Die transversale Ausdehnung im Kieferwinkelbereich wurde an den Styloiden orientiert. Nicht nur die Frontpartie, sondern auch die Korpusanteile bis in die Kieferwinkel wurden mit Schraubenlöchern versehen, um ggf. später doch noch Knochentransplantate fixieren zu können. Außerdem enthielten die Seitenflächen basal und kranial mit Fortsetzung entlang des Ramushinter- und -vorderrands je eine Reihe kleinerer Löcher in der Absicht, die Masseter/Pterygoideus-Schlinge und die Weichgewebe des Mundbodens (M. mylohyoideus) daran zu fixieren. Zur Oberflächenvergrößerung wurden posterior auf den Innenflächen des Titanunterkiefers kleine halbkugelige Höcker („Dots“) aufgebracht (Abb. 7h). Von Anfang an war klar, dass die alloplastische Neomandibula nicht zur kaufunktionellen Rehabiltation geeignet sein würde. Mit schleimhautgetragenen Prothesen auf den Titanelementen würden Ulcera und das Freilegen der Metalloberflächen drohen. Zum operativen Einbau der Neomandibula und der Fossae diente ein umlau- 


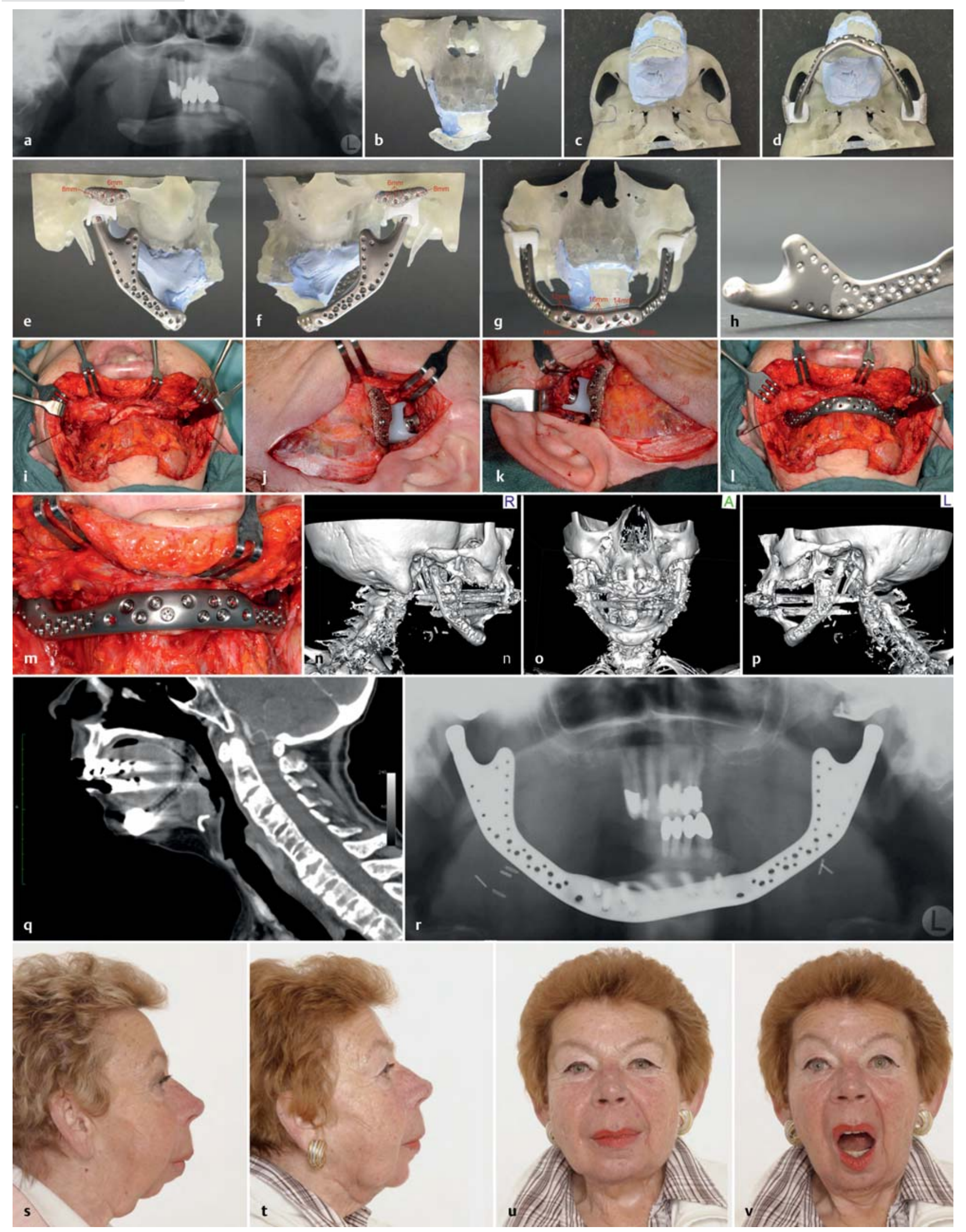

Legende Abb. 7 auf Seite 195 
Abb. 7a bis v TMJ-TJR beidseits - Neomandibula mit Fossakomponenten bei fortgeschrittener Osteolyse - a PSA: Symphyseales bzw. Kinn-Reststück des Unterkiefers nach 20-jährigem Krankheitsverlauf; b und c bei Planung am STL-Modell wird die Sollposition des anterioren Restunterkiefers mit zahnärztlichem Abdruckmaterial eingefroren; $\mathbf{d}$ bis $\mathbf{g}$ Design der Neomandibula, kurze Processus coronoidei, die nicht bis auf das Jochbogenniveau reichen; $\mathbf{h}$ Innenseite der Neomandibula mit Schraubenlöchern, Perforationen und „Dots“; $\mathbf{i}$ weite Exposition vom anterioren Unterkieferrest bis in die Nähe der leeren Gelenkgruben; $\mathbf{j}$ und $\mathbf{k}$ Sitz der Gelenkköpfe in den Fossakomponenten; I Ansicht der über die submandibuläre Route eingesetzten Neomandibula; über die Tunnelierung der Weichgewebe reichen die Kondylarfortsätze in die Fossae; m Schraubenfixierung des nach vorne und oben gezogenen Kinn-Reststücks am vorderen Bogen der Neomandibula; n 3-D-CTs 1 Woche postoperativ; q mediansagittale CT-Ebene - Hyoid angeschnitten, über suprahyoidale Muskelzüge nach vorne/oben aufgehängt, weit offener velopharyngealer Übergang; $\mathbf{r}$ PSA im Verlauf 3 Jahre postoperativ; s Gesichtsprofil lateral präoperativ; t Gesichtsprofil lateral und frontal 5 Jahre postoperativ; v Kieferöffnung auf $31 \mathrm{~mm}$ eingeschränkt; weitere Einzelheiten s. Fallbeschreibung im Text.

fender submandibulärer Zugang von Kieferwinkel zu Kieferwinkel in Kombination mit einer präaurikulär-temporalen Eröffnung beidseits. Der symphyseale Knochenrest wurde von basal und entlang der Vorderfläche freigelegt (Abb. 7i). Da Knochenoberflächen fehlten, entlang derer die Tunnelverbindungen zu den präaurikulär temporalen Wundkavitäten präpariert werden konnten, wurden die Alveolaris-inferior-Gefäß-Nervenbündel als Leitstrukturen benutzt. Jeweils im Mentalisbereich beginnend wurde lateral des neurovaskulären Bündels eine Route bis in die Infratemporalgrube geöffnet; die Gewebebrücke mit dem Fazialishauptstamm/-geflecht konnte dabei nach lateral abgedrängt werden. Im Anschluss an die Insertion der Fossakomponenten (Abb. 7j und k) wurde die Neomandibula eingepasst und die masseterico-pterygoidalen Weichgewebe adaptiert (Abb. 7I). Eine Befestigung der Temporalismuskeln glückte nicht. Sodann wurde das symphyseale Restknochenstück manuell nach vorne gezogen, an die Rückfläche des frontalen Neomandibulabogens angelagert und mit mehreren Schrauben fixiert (Abb. 7 m). Schließlich wurden die Mm. mylohyoidei seitlich vernäht und die Wunden schichtweise verschlossen. Im postoperativen Verlauf gab es keine besonderen Vorkomnisse. In der Nacht nach der Extubation am 2. Tag post OP schlief die Patienten erstmals seit Jahren wieder in Rückenlage, ohne Atemwegsverlegung und ohne Unterbrechungen. CT-morphologisch bestätigte sich die planmäßige Position aller Neomandibulakomponenten sowie des anterioren Unterkieferrests (Abb. 7n bis p) mit Resuspension der supra- bzw. infrahyoidalen Muskulatur und des Hyoids (Abb. 7q). In wiederholten Panoramaschichtaufnahmen findet sich erfreulicherweise bisher kein Hinweis auf eine fortschreitende Resorption des Unterkieferrests (Abb.7r). Mittlerweile liegt die Operation mehr als 5 Jahre zurück. Verglichen mit dem zurückliegenden Untergesicht im Ausgangsbefund (Abb. 7s) ist das seitliche Gesichtsprofil jetzt harmonisch und gerade (Abb. $7 \mathbf{t}$ und u). Die Patientin hat normale Atemund Schluckfunktion, die Kieferöffnung beträgt interinzisal ca. $31 \mathrm{~mm}$ und erfolgt ohne Deviationen nach lateral (Abb. 7v); Protusionsbewegungen sind nur angedeutet möglich. Ein Fremdkörpergefühl durch die Neomandibula wird nicht angegeben. Zu einer Exposition der Metalloberflächen ist es bisher nicht gekommen.

\section{Kinematik nach TMJ-TJRs-Endoprothesen}

Die Funktionsabläufe des Unterkiefers nach ein- oder beidseitigem alloplastischem Kiefergelenkersatz sind bisher nicht profund analysiert worden. Die Untersuchungen beschränken sich auf interinzisale Messungen ohne tiefere Einblicke in die Kinematik des Gelenks [42]. Mit der dynamischen Stereometrie lassen sich anatomische Daten aus der tomografischer Bildgebung (MRT, CT, DVT) mit der kinematischen Information aus einem optoelektronischen „Jaw“-Trackingsystem kombinieren und in Computersimulationen überlagern [43]. Mit dieser Technik kann die Bewegung frei wählbarer Punkte des Unterkiefers (Position, Geschwindigkeit und Beschleunigung) in einem zuvor definierten kartesischen Koordinatensystem betrachtet werden. Auf diese Weise kann in vivo die Kinematik des lateralen Gelenkkopf-Pols einer TMJ-TJR im Vergleich zum Bewegungsmuster im kontralateralen natürlichen Gelenk analysiert werden (Abb. 8). Wie nicht anders zu erwarten differieren die Bewegungspfade (Trajektorien) der lateralen Pole bei unilateralem TMJ-TJR erheblich. Bei Öffnungs- resp. Schließbewegungen des Unterkiefers (Abb.8a und b) findet am Kopf der Endoprothese vorwiegend eine Drehbewegung mit einer nur minimalen Translationskomponente statt. Die natürliche Gelenkwalze beschreibt hingegen die typische initiale Rotation, die in eine langstreckige S-förmige Translationsbewegung in der Sagittalebene übergeht. Bei der Unterkieferprotrusion ist der Bewegungsspielraum der TMJ-TJR in dorsoventraler und kraniokaudaler Richtung noch geringer als bei der Öffnung (Abb. 8c). Die Bewegungseinschränkungen in der TMJ TJR kommen in einer kontinuierlichen Deviation des Unterkiefers nach ipsilateral zum Ausdruck. Die kinematischen Bindungen mit reduzierten Freiheitsgraden für den artifiziellen Gelenkkopf sind durch die Konstruktionseigenschaften des UHMWPe-Gehäuses der Fossakomponente bedingt. Weitere Ursachen sind in der veränderten Weichgewebemechanik u.a. durch das Stripping der Insertion des M. pterygoideus lateralis zu suchen $[42,44]$. Bei bilateralem alloplastischen Gelenkersatz kann der Unterkiefer zwar weitgehend gerade geöffnet werden, die maximale Interinzisaldistanz, ebenso wie Protrusionsmöglichkeiten sind jedoch noch enger limitiert als nach Insertion eines unilateralen TMJ-TJR [45].

TMJ-TJRs können keine Normalfunktion wiederherstellen. Bei der Bewertung des Benefits für den Patienten muss vielmehr die Funktionsverbesserung in Bezug zur vorbestehenden Pathologie (Dysfunktion; Destruktion, Defekt) im Vordergrund stehen $[46,47]$.

\section{Infektionen nach alloplastischem Kiefergelenkersatz}

Postoperative Infektionen sind ein potenzielles Risiko nach einem alloplastischen Kiefergelenkersatz und stellen den Erfolg wesentlich infrage. In der weltweit vermutlich umfangreichsten Kohorte von 316 Patienten mit 579 TMJ Concepts Kiefergelenkendoprothesen war die Infektionsrate bei 2,5\% (bzw. 1,6\% bezogen auf die Zahl der Endoprothesen) angesiedelt [26]. Nach Angaben der 3 führenden TMJ-TJR-Herstellerfirmen bewegt sich die Infektionsrate ebenfalls zwischen 1,3 und $1,6 \%$.

Grundsätzlich sind akute von chronischen Infektionen zu unterscheiden.

Zu Frühinfekten kommt es unmittelbar postoperativ bzw. innerhalb von 3-4 Wochen. Spätinfekte treten in Zeiträu- 
men von mehr als 4-6 Wochen nach der Implantation der Endoprothese auf. Die Ursachen für eine Infektion einer TMJTJR-Endoprothese können in lokalen, perioperativen und systemischen Faktoren begründet sein. Eine bakterielle Kontamination mit Ausbildung eines Biofilms kann schon intraoperativ (unzureichende Sterilität, Kommunikation mit der Mundhöhle, Speichel, Nasensekret, Perforation des Gehörgangs) auftreten, sich später aus lokalen Quellen (Mundhöhle, Parodontitis, Nase, äußerer Gehörgang, Haaransatz, Haut) oder hämatogen (Lunge, Urogenitaltrakt) entwickeln. Das Risiko erhöht sich durch herabgesetzte Gewebequalität, Voroperationen, verminderte Vaskularisation, Diabetes, ein geschwächtes Immunsystem, Tabak- und Alkoholkonsum, Steroidmedikation, Immunsuppressiva, Fettleibigkeit und so weiter.

Mikrobielle Biofilme gelten als entscheidende Quelle für die Entstehung von Fremdmaterial- bzw. implantatassoziierten Infektionen. Im Unterschied zu Einzelbakterien in freier Suspension („planktonisches Wachstum“) sind die polybakteriellen Mikrokolonien in einer Mehrschichtmatrix aus polymeren Substanzen (extrazelluläre Polysaccharide) und Hydrogelen eingebettet und für Antibiotika kaum zugänglich. Häufig beteiligte Erreger im Multi-Spezies-Biofilm sind Staphylococcus aureus und epidermidis als auch Pseudomonas aeruginosa [48-50]. Die Antibiotikaresistenz der Bakterien im Biofilm kann um bis zu 3 Zehner-Potenzen höher liegen als im planktonischen Zustand. Zudem funktionieren die systemischen Abwehrmechanismen nicht, da die Entzündungsreaktion nur gering ausgeprägt ist und Antikörper nicht in der Lage sind, den Biofilm zu penetrieren [51].

Nur das physikalische Aufbrechen und die Entfernung der Biofilme durch extensives mechanisches Débridement und Wundspüllösungen bietet die Chance, die Wirkung von Antibiotika effektiver zu machen und die Kunstgelenke ggf. belassen zu können [52].

Die Behandlungsprotokolle bei Infektionen von Kunstgelenken verlangen daher ein aggressives Vorgehen. Die eingangs genannte Kohortenstudie [26] nennt folgende Erfolg versprechende Konzepte:

Bei akuten Infekten wird eine kalkulierte Breitspektrumantibitotikumtherapie begonnen und bis zum Erhalt von Punk-

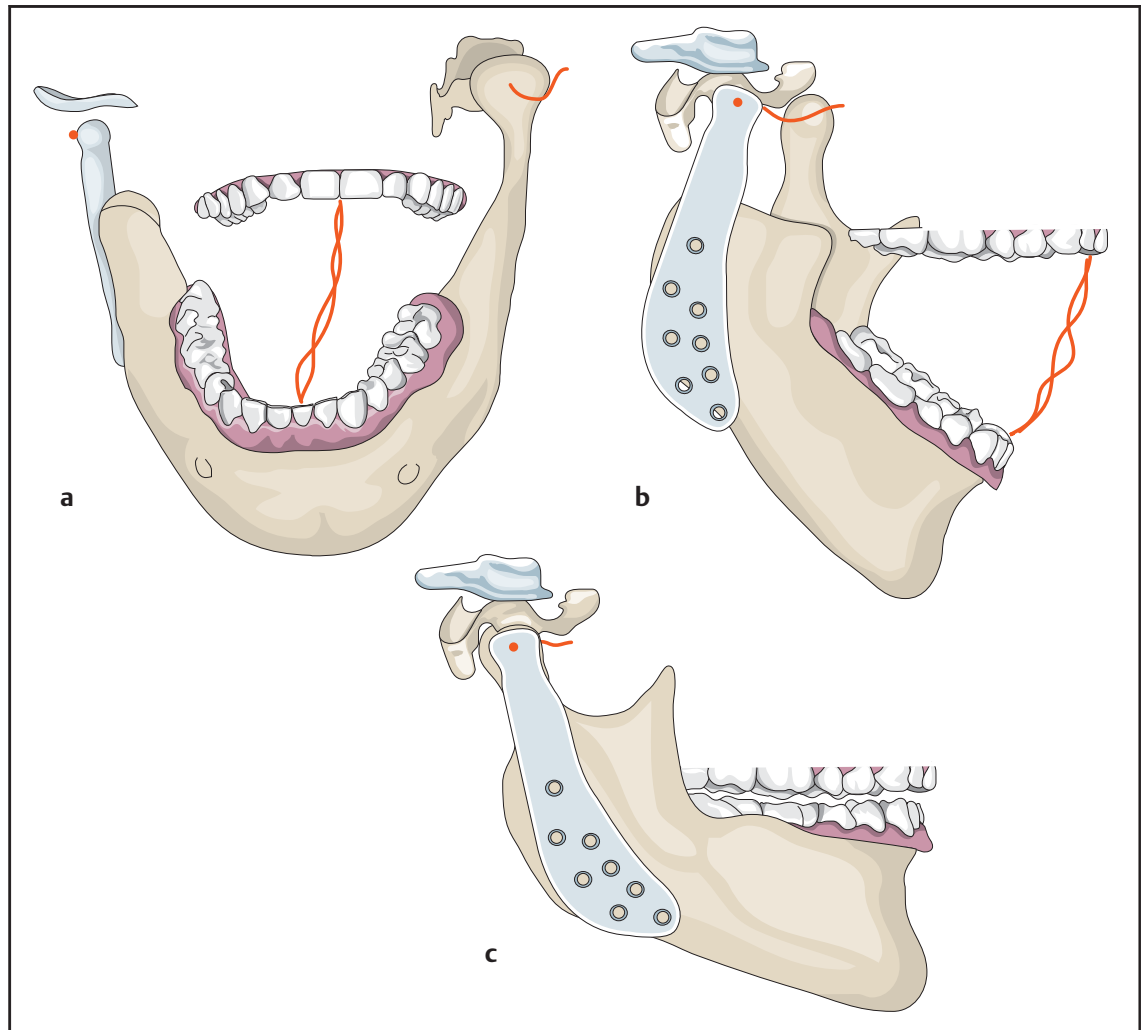

Abb. 8a bis c Dynamische Stereometrie bei unilateralem Kiefergelenkersatz mit einer TMJ-TJREndoprothese rechts - a Schrägansicht des Unterkiefer-Öffnungs-/Schließ-Zyklus, Bewegungspfade der lateralen Gelenkpole und des Interinzisalpunkts in rot; b Frontalansicht, kontinuierliche Deviation des Unterkiefers zur Seite des TMJ TJR angezeigt durch die Bewegungsbahn des Interinzisalpunkts. Ursache: kinematische Bindung des artifiziellen Gelenkkopfs; c Schrägansicht bei Protrusion: punktuelle Bewegung im TMJ TJR vs. Abwärts- und Vorwärts-Bewegungsbahn im natürlichen Gelenk (modifiziert nach [42]). Weitere Einzelheiten s. Text.

tions-/Abstrichergebnissen weitergeführt. Eine chirurgische Reintervention muss frühzeitig, vorzugsweise innerhalb von 5 Tagen nach Einsetzen der Symptomatik erfolgen. Zur Biofilmelimination wird ein sorgfältiges Débridement aller Oberflächen durch intensives Abschrubben mit einer sterilen Zahnbürste und Spülung mit Povidon-Iod-(PVP-Iod-)Lösung (Betaisodona ${ }^{\circledR}$ ) durchgeführt. Damit auch die Strukturen medial und dorsal der Fossa und des Gelenkschafts/kopfs erreicht werden können, muss der Gelenkkopf temporär luxiert werden. Danach werden 2 Spülkatheter und ein Penrose-Drain inseriert (Abb. 9) und die Wunde schichtweise verschlossen. Im 4Stunden-Takt werden dann während 45 Tagen über das geschlossene Kathetersystem Spülungen mit antibiotikahaltigen Lösungen vorgenommen. Nach Vorliegen von Kulturergebnissen und Antibiogramm wird eine gezielte i.v. Antibiotikatherapie über einen peripher gelegten (z.B. Armvenen), zentralen Venenkatheter (ZVK) gegeben, die auch nach Entlassung für weitere 4-6 Wochen fortgesetzt wird. Eine „second look“-OP bei klinisch infektfreien Verhältnissen und der Transfer vaskularisierten Gewebes (z.B. temporoparietaler Faszienlappen) werden zwar nicht beschrieben, sind aber insbesondere bei extendierten TMJ-TJRs sinnvolle Überlegungen.

Chronische Infekte haben eine schleichende Symptomatik. Die Patienten werden mit entzündlichen Lokalbefunden und Fistelbildungen im Bereich des äußeren Ohres, submandibulär oder im intermaxillären Mundschleimhautbezirk wieder vorstellig [53]. Ohne auf die Lockerung der Endoprothesenkomponenten (ja/nein) und etwaige Behandlungsalternativen einzugehen, wird zur Infektsanierung kompromisslos zum Ausbau der Implantate mit extensivem Débridement, Entfernung von Granulationsgewebe und Platzierung eines Katheterspülsystems geraten [26]. Das Antibiotikatherapie-Regime wird identisch gehandhabt wie bei akuten Infekten. Bei Konsolidierung und Infektfreiheit kann frühestens nach 8-10 Wochen ein neues TMJ TJR implantiert werden. Die Endoprothese wird dann mit einem freien au- 
tologen Fetttransplantat von abdominal umgeben [54,55].

\section{Diskussion, Zusammenfassung und Fazit}

Mit den Fortschritten der 3-D-CAD/CAMTechnologie, der computerassistierten Chirurgie und der Entwicklung biokompatibler Implantationsmaterialien haben die Sicherheit und das Indikationsspektrum für den alloplastischen Kiefergelenkersatz zugenommen. Trotzdem sind immer noch einige Diskussionspunkte offen sowohl für TMJ-TJR-Endoprothesen, die auf die Temporomandibularregion limitiert sind, als auch für in die Jochbogen/Jochbeinregion und/oder Unterkiefer extendierte Varianten.

Grundsätzlich sind autologe Rekonstruktionsverfahren gegenüber einem alloplastischen TMJ-TJR zu diskutieren [56].

Das traditionelle Verfahren für einen lokal limitierten Kiefergelenkersatz ist das nicht vaskularisierte kostochondrale Transplantat. Im direkten Vergleich mit einem alloplastischen Ersatz erfordern kostochondrale Transplantate höhere Reoperationsraten (Saeed et al. 2002) [57] bei höherer Komplikationshäufigkeit [58]. Wegen starker Rezidivneigung nach Anwendung im Anschluss an Ankyloseresektionen wird in dieser Indikation zu Zurückhaltung geraten [9].

Neben der kostochondralen Ersatzplastik sind zeitweise auch Distraktionsverfahren en vogue gewesen, z.B. in Form von Sliding-Osteotomien [6] bzw. einer Transportosteogenese [7] des Ramushinterrands nach kranial in die Fossa.

Als Optionen zum Kondylusersatz mit einem revaskularisierten Gewebetransfer sind das 2. Metarsophalangealgelenk [59] oder nach umfangreicheren Unterkieferteilresektionen mit einer Exartikulation v.a. Fibula Flaps beschrieben [60], in letzterer Situation auch myoossäre Flaps vom lateralen Skapularand. Das Ende des Fibulasegments für den Ramus/Kiefergelenkersatz wird üblicherweise abgerundet und in die Fossa glenoidalis eingesetzt. Insbesondere bei ausgedehnten Ersatzplastiken in Größenordnung der Hemimandibula oder mehr ist diese Position jedoch nicht immer stabil, und der Neokondylus nimmt wechselnde Stellungen zwischen Gelenkgrube und vor der Eminenz ein [6163]. Abhilfe, bis eine narbige Fixierung eintritt, lässt sich durch eine Aufhängung des Gelenkkopfs mit kräftigem

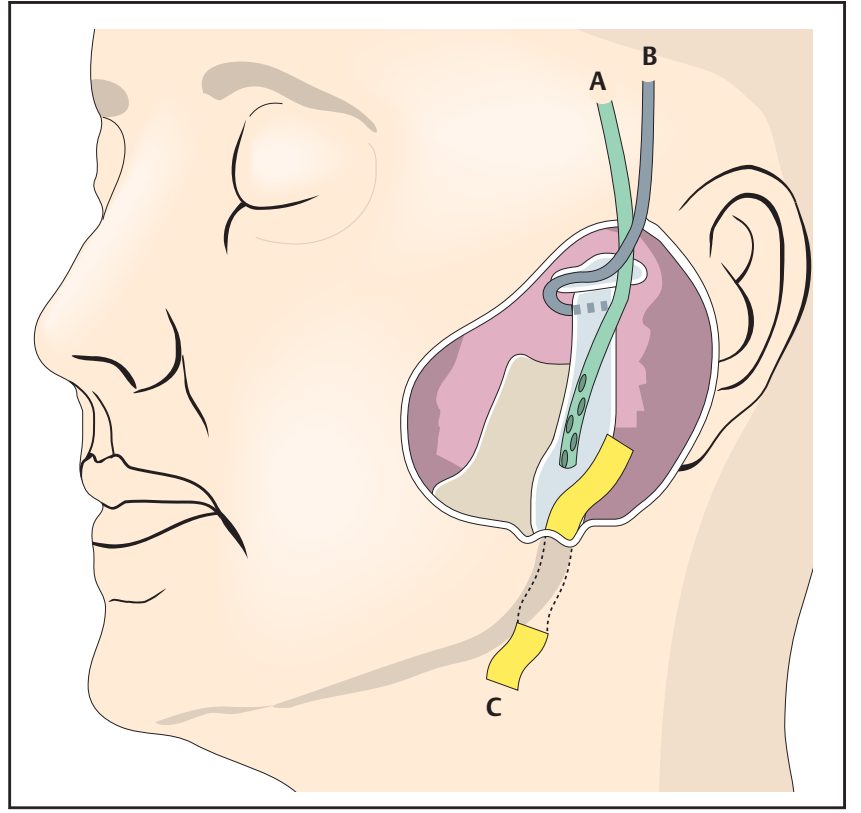

Abb. 9 Platzierung von 2 Spülkathetern und eines Penrose-Drains bei akuten Infektionen nach TMJ-TJR-Insertion - über separate Stichinzisionen präaurikulär wird ein Katheter auf der Lateralfläche der Unterkiefer-Ramus und der Schaftkomponente (A) eingeführt, der 2. Katheter auf der Medialseite (B) auf Höhe der artikulierenden Oberflächen von Gelenkkopf und UHMWPe-Gehäuse. Zum Ablauf der Spülflüssigkeit wird ein Penrose-Drain (C) von submandibulär auf der Lateralfläche des Ramus eingelegt (modifiziert nach Wolford et al. 2010 [26]).

nicht resorbierbaren Nahtmaterial am Diskus oder in der Temporalismuskulatur schaffen. Mehr Berechenbarkeit für eine eindeutige Kondylusposition und eine reproduzierbare (wenn auch limitierte) Gelenkkinematik bringt die Kombination von revaskularisiertem Knochentransfer und eines alloplastischen TMJ TJRs [64,65]. Solche Kombinationen sind in der Planung und in der operativ technischen Ausführung äußerst aufwendig und aufgrund ihrer Komplexität vermutlich fehleranfälliger als extendierte TMJ TJRs. Zur autologen Rekonstruktion eines vollständigen Unterkiefers kann es sogar notwendig werden, beide Fibulae $\mathrm{zu}$ entnehmen [66]. Im hier gezeigten Fall 2 bestand aufgrund der Gefäßsituation keine Alternative zur Versorgung mit einer alloplastischen Neomandibula.

Auch zur Notwendigkeit, TMJ TJRs längerfristig nur zusammen mit einer Fossakomponente $\mathrm{zu}$ verwenden, gibt es Gegenstimmen [67]. In einer Follow-upStudie mit einer mittleren Nachbeobachtungszeit von 7,8 Jahren fand sich in 131 Fällen, die nach Exartikulationen wegen eines Tumors oder Traumas eine Rekonstruktionsplatte mit einem Metallkondylus erhalten hatten, nur in 1 Fall eine Arrosion des äußeren Gehörgangs. Es wird jedoch betont, dass der Kondylus gegen den intakten Diskus oder gegen ein autologes (Temporalisfaszie, Sternocleidomastoideussehne) bzw. allogenes (Dura, Knorpel) Interface gesetzt wurde. Ein merklicher Vorteil individualisierter bzw. patientenspezifischer Implantate sind die technisch nahezu unbegrenzten Möglichkeiten zur Erweiterung und Anpassung an über die Gelenkregion hinaus bestehende Defekte. Patienten mit Gelenkfortsatzdefekten bei HFM wurden in den ersten Erfolgsberichten über extendierte TMJ-TJRs genannt $[1,9,68]$, wobei auf die anatomiegerechten 3-dimensionalen Rekonstruktionsmöglichkeiten hingewiesen wurde.

Voraussetzung zur erfolgreichen Insertion extendierter TMJ-TJR-Implantate ist ein gut vaskularisierter Weichgewebemantel mit ausreichender Resilienz. Eventuell muss der Implantation ein Gewebetransfer vorausgehen oder sie muss davon begleitet werden. Nach einer Bestrahlungstherapie ist eine TMJ-TJR-Insertion kritisch einzuschätzen und äußerste Zurückhaltung geboten.

Das Fehlen einer Masseter-PterygoidSchlinge, z. B. bei Entwicklungsdefiziten wie HFM oder nach Tumorresektionen, bringt gelegentlich das Problem von Luxationen des Metallgelenkkopfs aus der Fossa mit sich. Als Antwort darauf wurde von Herstellerseite das dorsale Schild des UHMWPe-Einsatzes des TMJ Con- 
cepts TJR verlängert und ist jetzt Standard. Zudem ist eine operative Zügelung sinnvoll, die man heute anstelle von Goretex - wie in Fall 1 - mit einem Streifen azellulärer dermaler Matrix oder Fascia lata vornehmen würde.

Eine weitere, seit Kurzem eingeführte Modifikation an der Schaftkomponente des TMJ Concepts TJR besteht in einer Öffnung direkt unterhalb des Gelenkkopfs in Anlehnung an die Fovea pterygoidea. Dieses Loch vereinfacht die vertikale Zügelung, in geeigneten Fällen kann auch der M. pterygoideus lateralis darin readaptiert werden.

Theoretisch erhöht sich mit zunehmender Länge der Schaft bzw. Mandibulakomponente extendierter TMJ-TJRs das Risiko für Instabilitäten, Materialversagen und Ermüdungsfrakturen. Tatsächlich wird die Dimensionierung der individualisierten Bauelemente so ausgelegt, dass sie weit über dem nicht biegbaren Abschnitt von Preformed Unterkieferrekonstruktionsplatten liegt, der sich nach bisherigen Erkenntnissen als äußerst resistent gegen Frakturen erwiesen hat [69].

Nach histologischen Untersuchungen zu urteilen, kommt es auch langfristig nicht zum Abrieb und daher in der Umgebung der artikulierenden Metallgelenkköpfe und der UHMWPe-Fossae nicht $\mathrm{zu}$ Fremdkörperreaktionen [70].

Abschließend bleibt festzuhalten - umfangreiche Primär- [71] und Sekundärrekonstruktionen des Unterkiefers, oder der Jochbogen/Jochbeinregion können nach computerassistierter Planung und Herstellung als extendierte patientenspezifische („patient fitting“) alloplastische Implantate zusammen mit einem Kiefergelenkersatz (TMJ-TJR) erfolgreich integriert werden. Wie die Literatur [72-74] und die beiden hier gezeigten Patientenbeispiele demonstrieren, sind dabei verlässliche Langzeitergebnisse $\mathrm{zu}$ erwarten.

\section{Literatur}

${ }^{1}$ Westermark A, Koppel D, Leiggener C. Condylar replacement alone is not sufficient for prosthetic reconstruction of the temporomandibular joint. Int J Oral Maxillofac Surg 2006; 35: 488-492

2 Freesmeyer WB. Zahnärztliche Funktionstherapie. München, Wien: Carl Hanser Verlag; 1993

${ }^{3}$ Yin CS, Lee YJ, Lee YJ. Neurological influences of the temporomandibular joint. J Bodyw Mov Ther 2007; 11: 285-294
${ }^{4}$ Khadka A, Hu J. Autogenous grafts for condylar reconstruction in treatment of TMJ ankylosis: current concepts and considerations for the future. Int J Oral Maxillofac Surg 2012; 41: 94-102

${ }^{5}$ Wolford LM, Cottrell DA, Henry C. Sternoclavicular grafts for temporomandibular joint reconstruction. J Oral Maxillofac Surg 1994; 52: 119-128; discussion 128-119

${ }^{6}$ Spagnoli DB, Gollehon SG. Distraction osteogenesis in reconstruction of the mandible and temporomandibular joint. Oral Maxillofac Surg Clin North Am 2006; 18: 383-398; vi

${ }^{7}$ Cheung LK, Lo J. The long-term effect of transport distraction in the management of temporomandibular joint ankylosis. Plast Reconstr Surg 2007; 119: 1003-1009

8 Saeed NR, Kent JN. A retrospective study of the costochondral graft in TMJ reconstruction. Int J Oral Maxillofac Surg 2003; 32: 606-609

9 Zanakis NS, Gavakos K, Faippea M et al. Application of custom-made TMJ prosthesis in hemifacial microsomia. Int J Oral Maxillofac Surg 2009; 38: 988-992

10 Westermark A, Heden P, Aagaard E et al. The use of TMJ Concepts prostheses to reconstruct patients with major temporomandibular joint and mandibular defects. Int J Oral Maxillofac Surg 2011; 40: 487-496

11 Lindqvist C, Soderholm AL, Hallikainen D et al. Erosion and heterotopic bone formation after alloplastic temporomandibular joint reconstruction. J Oral Maxillofac Surg 1992; 50: 942-949; discussion 950

12 Agaard E. Oral Presentation: Rationale for TMJ total joint replacement. International conference on TMJ biology, Diagnosis and Surgical Management. The Netherlands: University Medical Center Groningen; 2012 October $4-5$

13 Driemel O, Braun S, Muller-Richter UD et al. Historical development of alloplastic temporomandibular joint replacement after 1945 and state of the art. Int J Oral Maxillofac Surg 2009: 38: 909-920

14 van Loon JP, de Bont LG, Stegenga B et al. Groningen temporomandibular joint prosthesis. Development and first clinical application. Int J Oral Maxillofac Surg 2002; 31: 44-52

15 Kolk A, Sader R, Zeilhofer HF et al. Kollumrekonstruktion nach Resektion einer intrakapsularen Magenkarzinommetastase. Mund Kiefer Gesichtschir 2003; 7: 306-310

${ }^{16}$ Abramowicz S, Barbick M, Rose SP et al. Adaptability of stock TMJ prosthesis to joints that were previously treated with custom joint prosthesis. Int J Oral Maxillofac Surg 2012; 41: $518-520$

17 Vega LG, Gonzalez-Garcia R, Louis PJ. Reconstruction of acquired temporomandibular joint defects. Oral Maxillofac Surg Clin North Am 2013; 25: 251-269

18 Wolford LM, Pitta MC, Reiche-Fischel $O$ et al. TMJ Concepts/Techmedica custom-made TMJ total joint prosthesis: 5-year follow-up study. Int J Oral Maxillofac Surg 2003; 32: 268-274

19 Mercuri LG, Wolford LM, Sanders B et al. Custom CAD/CAM total temporomandibular joint reconstruction system: preliminary multicenter report. J Oral Maxillofac Surg 1995; 53: 106-115; discussion 115-116

20 Mercuri LG, Wolford LM, Sanders B et al. Longterm follow-up of the CAD/CAM patient fitted total temporomandibular joint reconstruction system. J Oral Maxillofac Surg 2002; 60: $1440-1448$

${ }^{21}$ Mercuri LG. Total joint reconstruction-autologous or alloplastic. Oral Maxillofac Surg Clin North Am 2006; 18: 399-410; vii
22 Pitta MC, Wolford LM. Use of acrylic spheres as spacers in staged temporomandibular joint surgery. J Oral Maxillofac Surg 2001; 59: 704-706

${ }^{23}$ Rasse M, Fialka V, Paternostro T. Modifikationen des Zugangs zum Kiefergelenk und Ramus mandibulae. Acta Chir Austriaca 1993; 25: 49-55

${ }^{24}$ Mercuri LG. Avoiding and managing temporomandibular joint total joint replacement surgical site infections. J Oral Maxillofac Surg 2012; 70: 2280-2289

${ }^{25}$ Wolford LM. Clinical indications for simultaneous TMJ and orthognathic surgery. Cranio 2007; 25: 273-282

${ }^{26}$ Wolford LM, Rodrigues DB, McPhillips A. Management of the infected temporomandibular joint total joint prosthesis. J Oral Maxillofac Surg 2010; 68: 2810-2823

27 Wolford LM, Dhameja A. Planning for combined TMJ arthroplasty and orthognathic surgery. Atlas Oral Maxillofac Surg Clin North Am 2011; 19: 243-270

28 Zizelmann C, Bucher P, Rohner D et al. Virtual restoration of anatomic jaw relationship to obtain a precise 3D model for total joint prosthesis construction for treatment of TMJ ankylosis with open bite. Int J Oral Maxillofac Surg 2010; 39: 1012-1015

29 Zizelmann C. Hammer B, Gellrich NC et al. An evaluation of face-bow transfer for the planning of orthognathic surgery. J Oral Maxillofac Surg 2012: 70: 1944-1950

${ }^{30}$ Roser M, Cornelius CP, Gehrke G et al. Osteotomien im Gesichtsskelett-Basis der Orthognathen Chirurgie: Befunderhebung, präoperative Planung und individuelle Realisation bei Korrekturoperationen von Kiefer- und Gesichtsdeformitäten. OP-Journal 2011; 27: 200-221

31 Mercuri LG, Edibam NR, Giobbie-Hurder A. Fourteen-year follow-up of a patient-fitted total temporomandibular joint reconstruction system. J Oral Maxillofac Surg 2007; 65: $1140-1148$

32 Cornelius $C P$, Ehrenfeld M, Schwenzer N. Zur Unterkieferrekonstruktion im Wachstumsalter. Fortschr Kiefer Gesichtschir 1994; 39: 141-146

33 Eckardt AM, Barth EL, Berten J et al. Pediatric mandibular resection and reconstruction: long-term results with autogenous rib grafts. Craniomaxillofac Trauma Reconstr 2010; 3: 25-32

34 Coleman SR. Structural Fat Grafting. St. Louis: Quality Medical Publishing; 2004

35 Coleman SR. "Facial augmentation with structural fat grafting". Clin Plast Surg 2006; 33 567-577

${ }^{36}$ Suei Y, Taguchi A, Tanimoto K. Diagnostic points and possible origin of osteomyelitis in synovitis, acne, pustulosis, hyperostosis and osteitis (SAPHO) syndrome: a radiographic study of 77 mandibular osteomyelitis cases. Rheumatology (Oxford) 2003; 42: 1398-1403

${ }^{37}$ McPhillips A, Wolford LM, Rodrigues DB. SAPHO syndrome with TMJ involvement: review of the literature and case presentation. Int J Oral Maxillofac Surg 2010; 39: 1160-1167

38 Salles M, Olive A, Perez-Andres R et al. The SAPHO syndrome: a clinical and imaging study. Clin Rheumatol 2011; 30: 245-249

39 Zemann W, Pau M, Feichtinger M et al. SAPHO syndrome with affection of the mandible: diagnosis, treatment, and review of literature. Oral Surg Oral Med Oral Pathol Oral Radiol Endod 2011; 111: 190-195

40 Niederdellmann $H$, Sadowy K, Reindl P. Der individuelle Unterkieferersatz nach ausgedehnten Kontinuitatsresektionen. Dtsch Z Mund Kiefer Gesichtschir 1988, 12: 330-332 
${ }^{41}$ Goncalves JR, Gomes LC, Vianna AP et al. Airway space changes after maxillomandibular counterclockwise rotation and mandibular advancement with TMJ Concepts ${ }^{\circledR}$ total joint prostheses: three-dimensional assessment Int J Oral Maxillofac Surg 2013; 42: 1014 1022

42 Leiggener CS, Erni S, Gallo LM. Novel approach to the study of jaw kinematics in an alloplastic TMJ reconstruction. Int J Oral Maxillofac Surg 2012; 41: 1041-1045

${ }^{43}$ Gallo LM. Modeling of temporomandibular joint function using MRI and jaw-tracking technologies-mechanics. Cells Tissues Organs 2005; 180: 54-68

${ }^{44}$ Celebi N, Rohner EC, Gateno J et al. Development of a mandibular motion simulator for total joint replacement. J Oral Maxillofac Surg 2011; 69: 66-79

45 Voiner J, Yu J, Deitrich P et al. Analysis of mandibular motion following unilateral and bilateral alloplastic TMJ reconstruction. Int J Oral Maxillofac Surg 2011; 40: 569-571

46 Linsen SS, Reich RH, Teschke M. Mandibular kinematics in patients with alloplastic total temporomandibular joint replacement-a prospective study. J Oral Maxillofac Surg 2012; 70: 2057-2064

47 Linsen SS, Reich RH, Teschke M. Pressure pain threshold and oral health-related quality of life implications of patients with alloplastic temporomandibular joint replacement-a prospective study. J Oral Maxillofac Surg 2012; 70: 2531-2542

48 Quinn PD, Giannakopoulos H, Carrasco L. Management of surgical failures. Oral Maxillofac Surg Clin North Am 2006; 18: 411-417; vii

49 Mercuri LG. Microbial biofilms: a potential source for alloplastic device failure. J Oral Maxillofac Surg 2006; 64: 1303-1309

${ }^{50}$ Mercuri LG. Avoiding and managing temporomandibular joint total joint replacement surgical site infections. J Oral Maxillofac Surg 2012; 70: 2280-2289

${ }^{51}$ Stewart PS, Costerton JW. Antibiotic resistance of bacteria in biofilms. Lancet 2001 358: $135-138$

${ }^{52}$ Costerton JW. Biofilm theory can guide the treatment of device-related orthopaedic infections. Clin Orthop Relat Res 2005: 7-11

${ }^{53}$ Hogan K, Herman A, Nicholson S et al. Late extrusion of a temporomandibular joint prosthesis masquerading as a sebaceous cyst J Plast Reconstr Aesthet Surg 2013; 66: e313-e314

54 Wolford LM, Karras SC. Autologous fat transplantation around temporomandibular joint total joint prostheses: preliminary treatment outcomes. J Oral Maxillofac Surg 1997; 55: 245-251; discussion 251-252

55 Wolford LM, Morales-Ryan CA, Morales PG et al. Autologous fat grafts placed around temporomandibular joint total joint prostheses to prevent heterotopic bone formation. Proc (Bayl Univ Med Cent) 2008; 21: 248-254

${ }^{56}$ Mercuri LG. Total joint reconstruction-autologous or alloplastic. Oral Maxillofac Surg Clin North Am 2006; 18: 399-410; vii

57 Saeed NR, Hensher R, McLeod MH et al. Reconstruction of the temporomandibular joint: autogenous compared with alloplastic. $\mathrm{Br}$ J Oral Maxillofac Surg 2002; 40: 296-299

58 Tang $W$, Long J, Feng $F$ et al. Condyle replacement after tumor resection: comparison of individual prefabricated titanium implants and costochondral grafts. Oral Surg Oral Med Oral Pathol Oral Radiol Endod 2009; 108: 147-152
${ }^{59}$ Bond SE, Saeed NR, Cussons PD et al. Reconstruction of the temporomandibular joint by the transfer of the free vascularised second metatarsal. Br J Oral Maxillofac Surg 2004; 42: 241-245

60 Potter JK, Dierks EJ. Vascularized options for reconstruction of the mandibular condyle. Semin Plast Surg 2008; 22: 156-160

${ }^{61}$ Engroff SL. Fibula flap reconstruction of the condyle in disarticulation resections of the mandible: a case report and review of the technique. Oral Surg Oral Med Oral Pathol Oral Radiol Endod 2005; 100: 661-665

62 Gonzalez-Garcia R, Naval-Gias L, RodriguezCampo FJ et al. Vascularized fibular flap for reconstruction of the condyle after mandibular ablation. J Oral Maxillofac Surg 2008; 66: 1133-1137

${ }^{63}$ Thor A, Rojas RA, Hirsch JM. Functional reconstruction of the temporomandibular joint with a free fibular microvascular flap. Scand J Plast Reconstr Surg Hand Surg 2008; 42: 233-240

64 Infante-Cossio P, Torres-Lagares D, Martinezde-Fuentes $R$ et al. Dental restoration with endosseous implants after mandibular reconstruction using a fibula free flap and TMJ prosthesis: a patient report. Int J Oral Maxillofac Implants 2006; 21: 481-485

65 Landes C, Korzinskas T, Dehner JF et al. Onestage microvascular mandible reconstruction and alloplastic TMJ prosthesis. J Craniomaxillofac Surg 2013; DOI: 10.1016/j. jcms.2013.01.043

66 Winters R, Saad A, Beahm DD et al. Total autogenous mandibular reconstruction using virtual surgical planning. J Craniofac Surg 2012; 23: e405-e407

${ }^{67}$ Marx RE, Cillo jr. JE, Broumand $V$ et al. Outcome analysis of mandibular condylar replacements in tumor and trauma reconstruction: a prospective analysis of 131 cases with long-term follow-up. J Oral Maxillofac Surg 2008; 66: 2515-2523

${ }^{68}$ Wolford LM, Bourland TC, Rodrigues D et al. Successful reconstruction of nongrowing hemifacial microsomia patients with unilateral temporomandibular joint total joint prosthesis and orthognathic surgery. J Oral Maxillofac Surg 2012; 70: 2835-2853

69 Probst FA, Schiel S, Cornelius CP et al. Vorgeformte winkelstabile Rekonstruktionsplatten zur Überbrückung von Defektzonen im Unterkiefer. OP-Journal 2012; 28: 188-192

70 Westermark A, Leiggener C, Aagaard E et al. Histological findings in soft tissues around temporomandibular joint prostheses after up to eight years of function. Int J Oral Maxillofac Surg 2011; 40: 18-25

${ }^{71}$ Ruiz Valero CA, Duran-Rodriguez G, SolanoParra $N$ et al. Immediate Total TMJ Replacement with TMJ Concepts ${ }^{\circledR}$ Prosthesis as an Alternative for Ameloblastoma cases. J Oral and Maxillofac Surg 2013; [Epub September 27]

72 Mercuri LG. A rationale for total alloplastic temporomandibular joint reconstruction in the management of idiopathic progressive condylar resorption. J Oral Maxillofac Surg 2007; 65: 1600-1609

73 Mehra P Wolford LM, Baran S et al. Singlestage comprehensive surgical treatment of the rheumatoid arthritis temporomandibular joint patient. J Oral Maxillofac Surg 2009; 67: 1859-1872

${ }^{74}$ Mercuri LG. Alloplastic temporomandibular joint replacement: rationale for the use of custom devices. Int J Oral Maxillofac Surg 2012; 41: 1033-1040
Prof. Dr. med. Dr. med. dent. Carl-Peter Cornelius

Oberarzt der Klinik

Prof. Dr. med. Dr. med. dent.

Michael Ehrenfeld

Direktor der Klinik

Dr. med. Dr. med. dent. Gerson Mast

Leitender Oberarzt der Klinik

Klinik und Poliklinik für Mund-,

Kiefer- und Gesichtschirurgie

Klinikum der Universität München

Ludwig-Maximilians-Universität

München

Lindwurmstr. 2a

80337 München

Peter.Cornelius@med-uni.muenchen.de

Prof. Anders Westermark, MD , DDS Consultant

OMFS/CMFS Surgery

Åland Central Hospital

AX 22111 Mariehamn

Åland

Finland

Priv.-Doz. Dr. med. Dr. med. dent. Oliver Driemel

Praxis für Mund-Kiefer-

Gesichtschirurgie

Steinburgsgang 2

26789 Leer (Ostfriesland)

Priv.-Doz. Dr. med. Dr. med. dent. Christoph Leiggener

Oberarzt

Klinik für Mund-, Kiefer- und

Gesichtschirugie

Universitätsspital Basel

Spitalstr. 1

4031 Basel

Schweiz 